Research Article

\title{
Contract Manufacturer's Encroachment Strategy considering Fairness Concern in Supply Chain
}

\author{
Jing Shi \\ School of Economics \& Management, Shanghai Maritime University, Shanghai 201306, China \\ Correspondence should be addressed to Jing Shi; jingshi@shmtu.edu.cn
}

Received 27 June 2020; Revised 30 August 2020; Accepted 12 January 2021; Published 16 February 2021

Academic Editor: Haipeng Peng

Copyright (C) 2021 Jing Shi. This is an open access article distributed under the Creative Commons Attribution License, which permits unrestricted use, distribution, and reproduction in any medium, provided the original work is properly cited.

\begin{abstract}
More and more contract manufacturers have started to establish their own brands besides providing manufacturing service for retailers who operate exclusive well-known brands. This paper studies the encroachment strategy of a contract manufacturer in a supply chain and the impact of fairness concern. Four scenarios are investigated by building game models: no fairness concern, the retailer's fairness concern, the contract manufacturer's fairness concern, and both members' fairness concern. The results show that when there is no fairness concern, the contract manufacturer always has motivation to encroach. However, when there exists fairness concern, only when the reservation price is sufficiently high, the contract manufacturer will encroach. Fairness concern has certain strength to stop the contract manufacturer's encroachment. When the reservation price is sufficiently low, the encroachment of the contract manufacturer benefits the retailer, or else it will harm the benefit of the retailer. The effect of fairness concern on profit margin and wholesale price decisions is opposite under different encroachment strategies. However, the fairness concern has no impact on the retail price of the private brand. Under encroachment strategy, contract manufacturer's or both members' fairness concerns have positive effect on the retailer's profit in certain conditions. However, the fairness concern always decreases the contract manufacturer's profit no matter what the form it is. Numerical examples show that it is best for the supply chain that both members have fairness concern under no encroachment. However, when the contract manufacturer has a private brand, it is best for the supply chain that no one has fairness concern when the advantageous inequity concern parameter is sufficiently low. When the advantageous inequity concern parameter is sufficiently high, it is best for the supply chain that both members have fairness concern.
\end{abstract}

\section{Introduction}

In past decades, outsourcing has played an important role in world economy [1-3]. There are a lot of contract manufacturers who provide production services for wellknown brands in China. The rapid development of e-commerce provides contract manufacturers with opportunities to sell directly to consumers. Many contract manufacturers have started to establish their own brands [4], which are called private brand [5], factory brand [6], or self-branded product [7]. For example, Sumvim has been the largest household textile company in China according to the value of export since 1999. In recent years, the company has established two private brands. One is Furi and the other is Jieyu, which are mainly sold in the domestic market in China. Recently, Sumvim has been cooperating with Pinduoduo Inc. (PDD) to promote her private brands while the latter has been one of the largest online group discounters in China. Actually, PDD launched the "New Brand Plan" at the end of 2018 aiming to hep 1000 manufacturers in China to establish their private brands. There are a lot of contract manufacturers adopting the same strategy as Sumvim, such as Guangdong Songfa Ceramics Co., Ltd., Zhejiang Sanhe Kitchenware Co., Ltd., and so on. However, there are still some manufacturers choosing not to build private brands, such as Crystal International Group Limited, who is a giant manufacturer of Uniqlo, H\&M, and Levi's [5]. This observation motivates us to study the encroachment strategy of contract manufacturers: encroachment or no encroachment. In what condition the contract manufacturer chooses to encroach and in what condition the 
manufacturer chooses not to encroach? What is the impact of encroachment on member's profit?

In making decision, people can be influenced by many factors. One important factor is fairness concern, which can be modelled as inequity aversion that people will sacrifice certain material payoff to achieve fairness [8]. Thus, an interesting question arises: whether fairness concern influences contract manufacturer's encroachment strategy? Different members may have different fairness concern $[9,10]$. Thus, this paper studies four different scenarios of fairness concern: (1) the retailer' fairness concern (scenario $A)$; (2) the contract manufacturer' fairness concern (scenario $B)$; (3) both the manufacturer and the retailer's fairness concern (scenario $C$ ); and (4) no fairness concern (scenario $N)$. The main questions this paper is trying to answer are as follows.

(1) What is the encroachment strategy of the contract manufacturer under different fairness concerns?

(2) What is the impact of encroachment on the profits of members?

(3) How do different fairness concerns impact the encroachment strategy of the contract manufacturer?

(4) How do different fairness concerns affect the decisions and profits of the contract manufacturer, the retailer, and the whole supply chain under two encroachment strategies?

This paper contributes to the literature of supply chain encroachment. Encroachment in supply chain has been a hot issue. The most studied encroachment in supply chain is the retailer's private label/store brand [11-15] and manufacturer's dual-channel strategy [16-19]. However, the contract manufacturer's encroachment has gained little attention. Several papers investigate the contract manufacturer's encroachment strategy. Shi [7] studied the quality decisions and the contract manufacturer's encroachment strategy under different channel leadership structures. The result shows that when the quality investment cost factor is sufficiently low, the contract manufacturer will never encroach. Sometimes, the encroachment of the contract manufacturer harms the benefit of the retailer. Cui [4] studied the free riding problem of quality considering the encroachment of the contract manufacturer. Chen et al. [5] explored the distribution channel decision of a contract manufacturer who operates a factory brand. However, to the best knowledge of the author, no paper has studied the impact of fairness concern on the encroachment strategy of contract manufacturer; this paper fills the gap; especially four different fairness concern scenarios are considered. Referring to retailer's encroachment, some results show that it will be a threat to the other member in the supply chain $[20,21]$, whereas in some condition, the other member will benefit from the encroachment of the retailer [22, 23]. Similarly, there are two different point of views about the manufacturer's dual-channel strategy. Some results show that adding a direct channel can harm the profit of the retailer; however, some studies show that it can be beneficial for the retailer in some instances [16-18]. This paper also finds that the contract manufacturer's encroachment is not always harmful to the other member. When the reservation price is sufficiently low, it will benefit the retailer, or else it will harm the retailer's profit. Li et al., [24] studied the impact of retailer's fairness concern on the supplier's encroachment. They find that when the retailer has fairness concern, the supplier has more flexibility to encroach and the retailer has more possibility to benefit from supplier encroachment. They also find that retailer's fairness concern can benefit the whole supply chain. Different from them, this paper considers not only the retailer's fairness concerns but also other two fairness concern scenarios. This paper finds that the retailer's fairness concern decreases the retailer's profit, whereas the contract manufacturer's fairness concern increases the retailer's profit. When both members have fairness concern, the impact on the retailer's profit can be negative or positive. The retailer's fairness concern improves the profit of the whole supply chain under no encroachment, whereas it decreases the profit of the whole supply chain under encroachment.

\section{Literature Review}

This paper relates to contract manufacturer's encroachment and fairness concern. This section will review related literature.

2.1. Contract Manufacturer's Encroachment. Supply chain encroachment is a very common phenomena in industry. Traditionally, there are two kinds of supply chain encroachment which are studied substantially. One is retailer's private brand/private label [11-15]. Giant retailers such as Walmart, Amazon, and JD.com all have private brands. They sell products for national manufacturers while at the same time sell products on their own brands in the same categories. Another type is manufacturer's direct channel/dualchannel strategy [16-19]. The manufacturer opens a direct channel selling the same product online or similar product under the same brand name, such as Apple and HP. However, the contract manufacturer's encroachment to build private brands/factory brands is rarely seen in literature. This research studies the encroachment strategy of the contract manufacturer: encroachment or no encroachment. Contract manufacturer's encroachment has gained little attention in the past. However, in recent years, there are more and more papers starting to pay attention to this issue $[4-7,25]$. Niu et al. [6] studied the impact of pricing sequence on the supply chain where the contract manufacturer owns a self-branded product besides providing manufacturing service to the client. Cui [4] combined the contract manufacturer's encroachment with quality decision where the contract manufacturer is a free rider who can imitate the client's product quality. Chen et al. [5] considered that a contract manufacturer introduces own-label product and competes with the client in the market. This own-label product is called factory brand in their paper. They mainly study the distribution problem of the factory brand. A three-level supply chain is considered. Shi [7] 
investigated the contract manufacturer's encroachment strategy considering quality and pricing decisions. Literature [25] extends literature [7] and considers the advertising effort. However, none of existing research has considered the fairness concern problem of members in the supply chain. This paper fills the gap. In above literature, the client who owns a well-known brand in the market is called original equipment manufacturer (OEM). However, in practice, the contract manufacturer who provides the manufacturing service is also called OEM. Thus, in order to avoid the confusion, this paper uses "retailer" to denote the client of the contract manufacturer. The retailer owns an existing well-known brand and focuses on the promotion of the brand. The contract manufacturer provides production for the retailer. Now the contract manufacturer has two strategies to choose from: encroachment or no encroachment.

2.2. Fairness Concern. There are mainly two kinds of fairness concerns studied in literature. One is peer-induced fairness concern and the other is distributional fairness concern. Peer-induced fairness considers the fairness in the horizontal level. For example, two retailers sell the products for a manufacturer. The retailer's fairness concerns about the other retailer is called peer-induced fairness. The retailer's fairness concerns about the manufacturer is called distributional fairness [9]. This paper only considers distributional fairness.

Fairness concern has been studied substantially in supply chain literature [10, 26-32]. Guan et al. [26] studied the channel coordination under Nash bargaining fairness concerns. Both quality improvement of the manufacturer and the advertising effort of the retailer are considered. Adhikari and Bisi [10] studied the impact of fairness concern on the greening and the pricing decisions in a green apparel supply chain. They considered two scenarios: the apparel retailer is fairness-concerned, whereas the apparel manufacturer is fairness-neutral and vice versa. They used Nash bargaining model to incorporate the fairness concern. Their results show that the greening quality and the retailer price are not affected by the retailer's fairness concern. However, it has a decreasing effect on the wholesale price under greening cost sharing contract. In profit sharing contract, the retailer's fairness concern lowers the greening quality, wholesale price, and the retail price, whereas the manufacturer's fairness concern does not have any impact on the decisions. Pan et al. [27] investigated a supply chain consisting of a dominant retailer and two manufacturers. The effects of fairness concern on pricing, profitability, and utility of supply chain members are discussed. Both peer-induced fairness concern and distributional fairness concern are considered. Sharma et al. [31] combined option contract with fairness concern by using Nash bargaining solution. Liu and Chen [33] studied a supply chain consisting of a dominated manufacturer and a newsvendor retailer. Each one of them can be inequity-averse. They studied the green level, wholesale price, and ordering quality of members. Li et al. [34] investigated a two-echelon supply chain containing a fairness-neutral manufacturer and a fairness- concerned retailer considering carbon emission reduction. However, they did not consider other fairness concern situations and supply chain encroachment.

Referring to combination of supply chain encroachment with fairness concern, there are very few studies. Huang and Feng [35] studied the retailer's encroachment strategy considering the manufacturer's fairness concern. For dualchannel setting where the manufacturer builds a direct channel selling the same product with traditional retail channel, there are a lot of studies considering the fairness concern problem. Li and $\mathrm{Li}$ [36] considered a dual-channel supply chain where a manufacturer adds a direct channel. The retailer has fairness concern and adds value-added service to the product. They find that channel efficiency falls with retailer's fairness concerns. Zhen et al. [9] studied a dual-supply chain in which a manufacturer produces and sells products through direct online channel and a retailer sells directly to consumers through online and offline channels. They considered four scenarios: no fairness concern, the retailer's fairness concern, the manufacturer' fairness concern, and both the manufacturer and the retailer have fairness concern. When referring to the contract manufacturer's encroachment, to the best knowledge of the author, there is no research about fairness concern problem. This paper contributes to literature by filling this gap.

2.3. Research Gap. This section discusses contribution to the existing literature. Comparison between this paper with relevant papers is shown in Table 1. There are several substantial contributions in this study. Firstly, there is substantial research on retailer's private brand (private-labelled products) and manufacturer's dual-channel strategy. However, there is little attention on the private brand strategy of the contract manufacturer who establishes a new brand selling directly to the end-consumer market besides providing manufacturing service for the retailer. Existing literature on contract manufacturer has not considered the fairness concern. This paper combines the contract manufacturer's encroachment strategy and fairness concern for the first time. Secondly, this paper compares the decisions and profit of the retailer and the contract manufacturer under four different member's fairness concerns: no fairness concern, retailer's fairness concern, contract manufacturer's fairness concern, and both members' fairness concern. Thirdly, this paper studies the impact of fairness concern on the encroachment strategy of the contract manufacturer.

\section{Basic Model}

Consider a supply chain consisting of a contract manufacturer and a well-known brand owner. In this paper, the brand owner is called as "retailer," such as IKEA, Nike, and Adidas. The contract manufacturer produces products for the retailer in OEM/ODM mode. That is, it is the retailer who sells the products to the end market. The contract manufacturer has no end-market brand. It is assumed that the retailer is the leader who has a stronger bargaining power than the contract manufacturer. The retailer moves first and 
TABLE 1: Comparisons between this paper with relevant papers $(N$ : no fairness concern; $A$ : retailer's fairness concern; $B$ : manufacturer's fairness concern; $C$ : both member's fairness concern; $R$ : retailer's encroachment; $M$ : manufacturer's encroachment with same brand; CM: contract manufacturer's encroachment with private brand).

\begin{tabular}{llllllll}
\hline Article & $R$ & $M$ & $C M$ & $N$ & $A$ & $B$ & $C$ \\
\hline Huang and Feng [35] & $\sqrt{ }$ & & & $\sqrt{ }$ & & $\sqrt{ }$ & \\
Li and Li [36] & & $\sqrt{ }$ & & & $\sqrt{ }$ & & \\
Li et al. [24] & & $\sqrt{ }$ & & & $\sqrt{ }$ & & \\
Arshad et al. [37] & & $\sqrt{ }$ & & & $\sqrt{ }$ & & \\
Zhang et al. [29] & & $\sqrt{ }$ & & & $\sqrt{ }$ & & \\
Adhikari and Bisi [10] & & & & $\sqrt{ }$ & $\sqrt{ }$ & $\sqrt{ }$ & \\
Pan et al. [27] & & & & & & & \\
Zhen et al. [9] & & $\sqrt{ }$ & & $\sqrt{ }$ & $\sqrt{ }$ & $\sqrt{ }$ & $\sqrt{ }$ \\
This paper & & $\sqrt{ }$ & $\sqrt{ }$ & $\sqrt{ }$ & $\sqrt{ }$ & $\sqrt{ }$ \\
\hline
\end{tabular}

demands a profit margin $m$, and then the contract manufacturer decides wholesale price $w$. The retail price in endconsumer market is $p=w+m$. Now the contract manufacturer is considering whether to establish a private brand of her own. Usually, the contract manufacturer will introduce a brand in the same category with the retailer's brand to fully utilize her production line. Thus, the private brand may compete with the retailer's existing brand in the consumer market. Usually, it is the retailer who gains most profit from the supply chain. When the supply chain structure experiences change, the profit of the supply chain is distributed in another way. Both members may rethink their decisions. In this paper, we incorporate fairness concern to their decisions to explore the impact of fairness concern on their decisions. This paper will study four different scenarios: neither the retailer nor the contract manufacturer has fairness concern $(N)$, the retailer has fairness concern $(A)$, the contract manufacturer has fairness concern $(B)$, or both have fairness concern $(C)$.

3.1. Demand Function. When the contract manufacturer encroaches, there are two brands in the market. One is the retailer's existing brand and the other is the contract manufacturer's private brand. According to the Hotelling model [38], the consumer market is conceptualized as a straight line. The retailer's existing brand and contract manufacturer's private brand are both located in the line. Consumers' preferences are uniformly distributed over the sufficiently long line with density one. This kind of assumption is commonly used in operations management literature [39-41]. The distance of consumer away from the brand indicates the preference of the brand, such as Nike and Adidas, McDonald's and KFC, and so on. In this paper, the retailer's existing brand and the contract manufacturer's private brand compete for consumers. Each consumer at most buys one unit. The distance of the two brands is $d$, which indicates brand differentiation $[39,40]$. A small $d$ means that two brands are highly substitutable, and vice versa. Assume that the distance of consumer away from brand $i$ is $x_{i}$ ( 1 indicates existing brand and 2 indicates contract manufacturer's private brand).
The utility of consumer from buying brand $i$ is

$$
U_{i}=r-p_{i}-t x_{i}
$$

where $r$ indicates the reservation price of consumer and $t$ reflects the transportation cost (or disutility), which indicates the intensity of consumer's preference for a brand. A consumer buys the product only when the consumer can get non-negative utility $\left(U_{i} \geq 0\right)$. In this paper, the production cost of two brands is the same since they are both produced by the contract manufacturer. Let $c$ denote the production cost of two brands. When the retail price is equal to the production cost, the utility of the customer in the middle point must be non-negative, i.e., $r>c+(t d / 2)$, or else two brands will not compete. A greater $t$ means a higher loyalty.

When two brands compete, the consumer who locates in the non-differentiation point gets the same utility from two brands, that is: $r-p_{1}-t x_{1}=r-p_{2}-t\left(d-x_{1}\right)$. Solving this equation for $x_{1}$, we can get $\bar{x}_{1}=\left(\mathrm{d} t-p_{1}+p_{2}\right) /(2 t)$. Solving $U_{i}=0$ for $x_{i}$, we can obtain the demand of the loyal consumers for brand $i$. Following $\mathrm{Xu}$ et al. [40] and Xiao et al. [42], when the existing brand and the contract manufacturer's private brand compete, we can obtain the demand rate for brand $i$ during a period.

$$
q_{i}=\bar{x}_{i}+\frac{r-p_{i}}{t}=\frac{2 r+\mathrm{d} t-3 p_{i}+p_{j}}{2 t}, \quad i, j=1,2, j \neq i .
$$

The market scale $(2 r+\mathrm{d} t) /(2 t)$ is a decreasing function of transportation cost $t$ and an increasing function of the reservation price $r$ or the brand differentiation $d$.

When the contract manufacturer chooses not to encroach, there is only one brand in the market. If the utility of the consumer is non-negative, consumers will buy the product from the retailer, i.e., $r-p-t x>0$, where $p=w+m$.

Thus, the demand of the retailer is

$$
\bar{q}=\frac{2(r-p)}{t} \text {. }
$$

\subsection{The Optimization Problem of the Retailer and the Contract Manufacturer}

3.2.1. Scenario N: When No Member Has Fairness Concern. We use suffix " $N N$ " to indicate the scenario when the retailer has fairness concern under no encroachment. We use suffix " $N E$ " to indicate the scenario when the retailer has fairness concern under encroachment.

When no member has fairness concern, the profit of the contract manufacturer and the retailer is as follows. We use subfix " $M$ " and " $R$ " to indicate the contract manufacturer and the retailer, respectively.

$$
\begin{aligned}
& \pi_{M}^{N N}=(w-c) \bar{q}, \\
& \pi_{R}^{N N}=m \bar{q} .
\end{aligned}
$$


When the contract manufacturer encroaches, the profit functions of the retailer and the contract manufacturer are as follows.

$$
\begin{aligned}
& \pi_{M}^{N E}=(w-c) q_{1}+\left(p_{2}-c\right) q_{2} \\
& \pi_{R}^{N E}=m q_{1}
\end{aligned}
$$

where $q_{1}$ and $q_{2}$ are shown in equation (2).

By using backward induction, we can get the equilibrium profits of both players as $\pi_{M}^{N N *}=(2 r+\mathrm{d} t-2 c)^{2} /(48 t)$, $\pi_{R}^{N N *}=(r-c)^{2} /(4 t), \quad \pi_{M}^{N E *}=3(2 r+\mathrm{d} t-2 c)^{2} /(32 t)$, and $\pi_{R}^{N E *}=(r-c)^{2} /(8 t)$. The proof can be found in Appendix (Proof of Proposition 1).

3.2.2. Scenario A: When the Retailer Has Fairness Concern. Following Nie and Du [43], Cui et al. [44], and Zhen et al. [9], we adopt utility function as the retailer's optimization objection. Fehr and Schmidt [8] state that positive benefit and negative benefit of fairness concerns both cause losses to their utility. Thus, in this paper, we both consider disadvantageous inequality and advantageous inequity. We use suffix " $A N$ " to indicate the scenario when the retailer has fairness concern under no encroachment. Suffix " $A E$ " indicates the scenario when the retailer has fairness concern under encroachment.

Under no encroachment, the utility function of the retailer is

$$
U_{R}^{A N}=\pi_{R}^{N N}-\alpha\left(\pi_{M}^{N N}-\pi_{R}^{N N}\right)^{+}-\beta\left(\pi_{R}^{N N}-\pi_{M}^{N N}\right)^{+} .
$$

The objective function of the contract manufacturer does not change, i.e., $\pi_{M}^{A N}=\pi_{M}^{N N}$. is

Under encroachment, the utility function of the retailer

$$
U_{R}^{A E}=\pi_{R}^{N E}-\alpha\left(\pi_{M}^{N E}-\pi_{R}^{N E}\right)^{+}-\beta\left(\pi_{R}^{N E}-\pi_{M}^{N E}\right)^{+} .
$$

The objective function of the contract manufacturer does not change, i.e., $\pi_{M}^{A E}=\pi_{M}^{N E}$. $\alpha$ and $\beta$ denote the disadvantageous inequity and advantageous inequity concern parameters, respectively, where $\alpha>\beta$ and $0<\beta<1$ [9]. If the retailer's profit is lower than the equitable payoff, a disadvantageous inequity occurs, resulting in disutility for the retailer. However, if his profit is higher than the equitable payoff, an advantageous inequity also occurs. The greater $\alpha$ (or $\beta$ ) is, the more the retailer is concerned about the disadvantageous (or advantageous) inequity. $\alpha>\beta$ means that the impact of the disadvantageous inequity is higher than that of the advantageous inequity.

Based on the obtained equilibrium profits of the contract manufacturer and the retailer in scenario $N$ (without fairness concerns), when the contract manufacturer does not encroach, the profit of the retailer is higher than that of the contract manufacturer. However, when the contract manufacturer encroaches, the profit of the retailer is lower than that of the contract manufacturer.

Thus, equations (6) and (7) can be rewritten as

$$
\begin{aligned}
& U_{R}^{A N}=\pi_{R}^{N N}-\beta\left(\pi_{R}^{N N}-\pi_{M}^{N N}\right), \\
& U_{R}^{A E}=\pi_{R}^{N E}-\alpha\left(\pi_{M}^{N E}-\pi_{R}^{N E}\right) .
\end{aligned}
$$

\subsubsection{Scenario B: When the Contract Manufacturer Has} Fairness Concern. Similarly, we use suffix " $B N$ " to denote the scenario when the contract manufacturer has fairness concern under no encroachment. We use suffix " $B E$ " to denote the scenario when the contract manufacturer has fairness concern under encroachment.

Similar to scenario $A$, under no encroachment, the utility function of the contract manufacturer is

$$
U_{M}^{B N}=\pi_{M}^{N N}-\alpha\left(\pi_{R}^{N N}-\pi_{M}^{N N}\right) .
$$

The objective function of the retailer does not change, that is, $\pi_{R}^{B N}=\pi_{R}^{N N}$.

Under encroachment, the utility function of the contract manufacturer is

$$
U_{M}^{B E}=\pi_{M}^{N E}-\beta\left(\pi_{M}^{N E}-\pi_{R}^{N E}\right)
$$

The objective function of the retailer does not change, that is, $\pi_{R}^{B E}=\pi_{R}^{N E}$.

3.2.4. Scenario C: Both the Retailer and the Contract Manufacturer Have Fairness Concern. In this section, we use suffix " $C N$ " to denote the scenario when the retailer and the contract manufacturer both have fairness concern under no encroachment. We use suffix " $C E$ " to denote the scenario when the retailer and the contract manufacturer both have fairness concern under encroachment.

Similar to scenario $A$, under no encroachment, the utility function of the retailer and the contract manufacturer is as follows.

$$
\begin{aligned}
& U_{M}^{C N}=\pi_{M}^{N N}-\alpha\left(\pi_{R}^{N N}-\pi_{M}^{N N}\right), \\
& U_{R}^{C N}=\pi_{R}^{N N}-\beta\left(\pi_{R}^{N N}-\pi_{M}^{N N}\right) .
\end{aligned}
$$

Under encroachment, the utility function of the retailer and the contract manufacturer is as follows.

$$
\begin{aligned}
& U_{M}^{C E}=\pi_{M}^{N E}-\beta\left(\pi_{M}^{N E}-\pi_{R}^{N E}\right), \\
& U_{R}^{C E}=\pi_{R}^{N E}-\alpha\left(\pi_{M}^{N E}-\pi_{R}^{N E}\right) .
\end{aligned}
$$

Under each scenario, the time sequence of the game is as follows.

(i) At the first stage, the contract manufacturer decides whether to encroach.

(ii) At the second stage, the retailer decides profit margin.

(iii) At the third stage, the contract manufacturer decides wholesale price. If she decides to encroach, she also needs to decide the retail price of the private brand.

The list of notations is shown in Table 2. 
TABle 2: Notations.

\begin{tabular}{|c|c|}
\hline Parameter & The meaning of the parameter \\
\hline$\alpha$ & Disadvantageous inequity concern parameter $0<\alpha<1$ \\
\hline$\beta$ & Advantageous inequity concern parameter $0<\beta<1, \alpha>\beta$ \\
\hline$d$ & Brand differentiation \\
\hline$t$ & Transportation cost (or disutility), which indicates the intensity of consumer's preference for a brand \\
\hline$q_{i}$ & Demand rate for brand $i$ when the contract manufacturer encroaches \\
\hline $\bar{q}$ & Demand of the retailer when the contract manufacturer does not encroach \\
\hline$m^{i N},(i=N, A, B, C)$ & Retailer's profit margin under no encroachment \\
\hline$w^{i N},(i=N, A, B, C)$ & Contract manufacturer's wholesale price under no encroachment \\
\hline$p_{2}^{i N},(i=N, A, B, C)$ & Retail price of the contract manufacturer's private brand under no encroachment \\
\hline$\pi_{M}^{i N}, \pi_{R}^{i N},(i=N, A, B, C)$ & Profits of the contract manufacturer and the retailer in scenario $i$ under no encroachment \\
\hline$\pi_{T}^{i N},(i=N, A, B, C)$ & Profits of the whole supply chain in scenario $i$ under no encroachment \\
\hline$m^{i E},(i=N, A, B, C)$ & Retailer's profit margin under encroachment \\
\hline$w^{i E},(i=N, A, B, C)$ & Contract manufacturer's wholesale price under encroachment \\
\hline$p_{2}^{i E},(i=N, A, B, C)$ & Retail price of contract manufacturer's private brand under encroachment \\
\hline$\pi_{M}^{i E}, \pi_{R}^{i E},(i=N, A, B, C)$ & Profits of the contract manufacturer and the retailer in scenario $i$ under encroachment \\
\hline$\pi_{T}^{i E},(i=N, A, B, C)$ & Profits of the whole supply chain in scenario $i$ under encroachment \\
\hline
\end{tabular}

\section{Equilibrium}

Firstly, the equilibrium in each scenario can be derived by backward induction.

Proposition 1. The equilibriums in scenario $N, A, B$, and $C$ are summarized in Table 3.

In order to ensure the positivity of the utility, $G \geq 0$ must be satisfied. From Proposition 1, the retail price of the incumbent brand is $p_{1}^{N E *}=m^{N E *}+w^{N E *}=(2 r+$ $\mathrm{d} t+c) / 3$. Then, we can get $p_{1}^{N E *}-p_{2}^{N E *}=(2 r+\mathrm{d} t-2 c) /$ $12>0$ because $r>c$ (the reservation price is higher than the production cost). That is to say, the retail price of the new brand is lower than the price of the retailer's existing brand, which is consistent with practice. Usually, the contract manufacturer's private brand is more affordable compared with the retailer's brand. From Proposition 1, we can get Proposition 2.

Proposition 2. The profits of the retailer and the contract manufacturer have following properties.

(i) $\pi_{R}^{N N *}=2 \pi_{M}^{N N *} ; \pi_{M}^{N E *}=4.5 \pi_{R}^{N E *}$.

(ii) $\pi_{R}^{A N *}=(2-3 \beta) \pi_{M}^{A N *} ; \quad U_{R}^{A E *}=k(\alpha) \pi_{M}^{A E *}$, where $k(\alpha)=\left((2+3 \alpha)\left(1-2 \alpha-5 \alpha^{2}\right) /\left(9+26 \alpha+19 \alpha^{2}\right)\right)$.

(iii) $U_{M}^{B N *}=((1 / 2)+\alpha) \pi_{R}^{B N *} ; \pi_{R}^{B E *}=\left(2 /(9-18 \beta) U_{M}^{B E *}\right)$.

(iv) $U_{R}^{C N *}=l(\alpha) U_{M}^{C N *}$, where $l(\alpha)=([2+2 \alpha(1-\beta)-$ $3 \beta] /[(1+2 \alpha)(1+\alpha)]) ; U_{M}^{C E *}=v(\alpha) U_{R}^{C E *}$, where $v$ $(\alpha)=((1-2 \beta)(1-\beta) G /\{[2+3 \alpha-2(1+\alpha) \beta] H\})$.

Proposition 2 (i) shows that the retailer's profit is twice the contract manufacturer's profit. The retailer who owns a well-known brand shares more profit of the supply chain than the contract manufacturer, which are consistent with practice. The reason is that the retailer leads the supply chain and moves first. There are first-mover advantages [9]. When the contract manufacturer establishes her private brand, she can get more profit than the retailer. The retailer may feel unfair. As a result, the retailer may stop cooperating with the contract manufacturer. Thus, the contract manufacturer may need to consider fairness concern.

Proposition 2 (ii) shows that when $\beta$ increases, the difference between profits of both members will become smaller. That is, when the advantageous inequity concern parameter increases, the share of profit in supply chain is more balanced in Model AN. Because $(\mathrm{d} k(\alpha) / \mathrm{d} \alpha)=-(\{61+$ $\left.\alpha\{364+\alpha[802+15 \alpha(52+19 \alpha)]\}\} /[9+\alpha(26+19 \alpha)]^{2}\right)<0$, $k(\alpha)$ is a decreasing function of $\alpha$. In scenario $A$, it is the retailer who has fairness concern. When the disadvantageous inequity concern parameter $\alpha$ increases, the share of profit between members will be more imbalanced, which is opposite to the situation when the contract manufacturer does not encroach. That is, the fairness concern of the retailer will decrease his utility. The reason is that it is the contract manufacturer who gains more profit of the supply chain when the contract manufacturer encroaches. If the retailer wants to get more profit, whether the contract manufacturer has fairness concern is more important.

From Proposition 2 (iii), when the disadvantageous inequity $\alpha$ increases, the contract manufacturer can get more profits. That is, the fairness concern increases the profit of the contract manufacturer. The share of profit is more balanced.

\section{The Encroachment Strategy of the Contract Manufacturer}

In this section, we will discuss the contract manufacturer's encroachment strategy when neither the retailer nor the contract manufacturer has fairness concern. Only when the contract manufacturer can get more profit from encroachment, she will encroach. By comparing the profits of the contract manufacturer under two different encroachment strategies, we can get Proposition 3.

Proposition 3. In different scenarios, the encroachment strategies are different. 


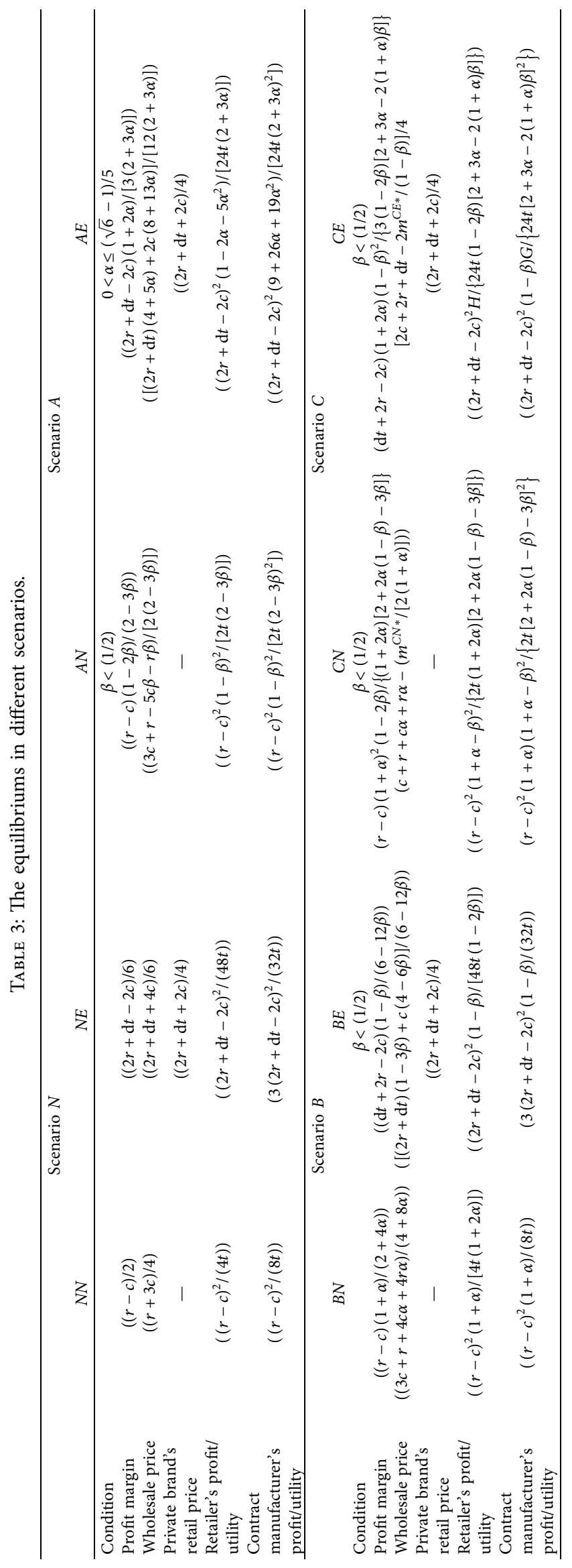


(i) In scenario $N$, when no member in the supply chain has fairness concern, the contract manufacturer will always encroach.

(ii) In scenario $A$, only the retailer has fairness concern. When $r \geq([2 c L-2 \sqrt{3} c(2+3 \alpha)(1-\beta)-d t L] /[2 L-$ $2 \sqrt{3}(2+3 \alpha)(1-\beta)])$, the contract manufacturer will encroach, or else she will not encroach, where $L=\sqrt{9+\alpha(26+19 \alpha)}(2-3 \beta)$.

(iii) In scenario $B$, only the contract manufacturer has fairness concern. When $r \geq([2 c(2+\alpha-3 \beta)-$ $d t(3+\sqrt{3(1-\alpha)} \sqrt{1-\beta}-3 \beta)] /[2(2+\alpha-3 \beta)])$, the contract manufacturer will encroach, or else she will not encroach.

(iv) In scenario $C$, both the retailer and the contract manufacturer have fairness concern. When $r \geq((2 c J-d t J-c K) /(2 J-K))$, the contract manufacturer will encroach, or else she will not encroach, where $J=[2+2 \alpha(1-\beta)-3 \beta] \sqrt{1-\beta} \sqrt{G}$ and $K=2 \sqrt{3} \sqrt{1+\alpha}(1+\alpha-\beta)[2+3 \alpha-2(1+\alpha) \beta]$.

Proposition 3 (i) shows that if both retailer and the contract manufacturer do not have fairness concern, the contract manufacturer will always encroach. Selling directly to end-market consumers are more beneficial to the contract manufacturer when no one has fairness in the supply chain. Proposition 3 (ii)-(iv) show the conditions that the contract manufacturer will encroach under different scenarios. When the reservation price is sufficiently high, the contract manufacturer has the motivation to encroach, or else she will not encroach. From Proposition 3, fairness concern has certain strength to stop the contract manufacturer to encroach.

Next, the impact of encroachment on the retailer is analyzed as follows.

In the following, we assume that the equilibrium profit is equal to utility in each scenario to make the expressions simplified. That is, $\pi_{R}^{A N *}=U_{R}^{A N *}, \pi_{R}^{C N *}=U_{R}^{C N *}, \pi_{M}^{B N *}=$ $U_{M}^{B N *}, \pi_{M}^{C N *}=U_{M}^{C N *}, \quad \pi_{R}^{A E *}=U_{R}^{A E *}, \pi_{R}^{C E *}=U_{R}^{C E *}, \pi_{M}^{B E *}$ $=U_{M}^{B E *}, \pi_{M}^{C E *}=U_{M}^{C E *}$.

Proposition 4. The impact of contract manufacturer's encroachment on the retailer is as follows.

(i) Under scenario $N$, when $r>(c+d t /(2 \sqrt{3}-2))$, $\pi_{R}^{N N *}>\pi_{R}^{N E *}$, or else $\pi_{R}^{N N *} \leq \pi_{R}^{N E *}$.

(ii) Under scenario $A$, when $r>((d t-2 c) N /(1-2 N))$, $\pi_{R}^{A N *}>\pi_{R}^{A E *}$, or else $\pi_{R}^{A N *} \leq \pi_{R}^{A E *}$, where $N=\sqrt{\left(\left(1-2 \alpha-5 \alpha^{2}\right)(2-3 \beta) /\left[12(1-\beta)^{2}(2+3 \alpha)\right]\right)}$.

(iii) Under scenario $B, \quad r>(c+d t /(1-2 V))$, $\pi_{R}^{B N *}>\pi_{R}^{B E *}$, or else $\pi_{R}^{B N *} \leq \pi_{R}^{B E *}$, where $V=\sqrt{((1-\beta)(1+2 \alpha) /[14(1+\alpha)(1-2 \beta)])}$.

(iv) Under scenario $C$, when $r>(c+d t /(1-2 Z))$, $\pi_{R}^{C N *}>\pi_{R}^{C E *}$, or else, $\pi_{R}^{C N *} \leq \pi_{R}^{C E *}$, where $Z=\sqrt{(H(1+2 \alpha)[2+2 \alpha(1-\beta)-3 \beta] /\{12}$ $\left.\left.(1+\alpha-\beta)^{2}(1-2 \beta)[2+3 \alpha-2(1+\alpha) \beta]\right\}\right)$.
Proposition 4 shows that when the reservation price is sufficiently low, the encroachment of the contract manufacturer benefits the retailer, or else it will harm the benefit of the retailer. That is, the encroachment of the contract manufacturer does not always harm the retailer. The results are similar across four different fairness concern scenarios. Proposition 3 shows the condition that the contract manufacturer will encroach. Thus, the analysis of impact of encroach on the contract manufacturer is omitted.

\section{The Impact of Fairness Concern}

In this section, we will study the impact of fairness concerns on decisions and profits. Firstly, we will compare the equilibriums in four scenarios $(N, A, B, C)$ under no encroachment. Then, we will compare the equilibriums under encroachment. Finally, we will use numerical examples to illustrate the impact of several key parameters.

Proposition 5. Under no encroachment,

(i) $m^{A N *}<m^{N N *}, m^{B N *}<m^{N N *}, m^{C N *}<m^{N N *}$; when $0<\beta \leq(2 \alpha /(1+5 \alpha)), \quad m^{B N *} \leq m^{A N *} ;$ when $(2 \alpha /$ $(1+5 \alpha))<\beta<(1 / 2), m^{B N *}>m^{A N *} ; m^{C N *}<m^{A N *}$; $m^{C N *}<m^{B N *}$.

(ii) $w^{A N *}>w^{N N *} ; w^{B N *}>w^{N N *} ; w^{C N *}>w^{N N *} ;$ when $0<\beta<(4 \alpha /(1+8 \alpha)), w^{B N *}>w^{A N *}$. When $(4 \alpha /(1+$ $8 \alpha)) \leq \beta<(1 / 2), \quad w^{B N *} \leq w^{A N *} ; \quad w^{C N *}>w^{A N *} ;$ $w^{C N *}>w^{B N *}$.

(iii) $\pi_{R}^{A N *}<\pi_{R}^{N N *} ; \pi_{R}^{B N *}<\pi_{R}^{N N *} ; \pi_{R}^{C N *}<\pi_{R}^{N N *} ;$ if $\alpha<$ $(1 / 7)$, when $\left(\left(1+5 \alpha-\sqrt{1-6 \alpha-7 \alpha^{2}}\right) /[4(1+2 \alpha)]\right)<$ $\beta<\left(\left(1+5 \alpha+\sqrt{1-6 \alpha-7 \alpha^{2}}\right) /[4(1+2 \alpha)]\right), \pi_{R}^{B N *}>\pi_{R}^{A N *}$; when $\beta \leq\left(\left(1+5 \alpha-\sqrt{1-6 \alpha-7 \alpha^{2}}\right) /[4(1+2 \alpha)]\right)$ or $\beta \geq\left(\left(1+5 \alpha+\sqrt{1-6 \alpha-7 \alpha^{2}}\right) /[4(1+2 \alpha)]\right), \quad \pi_{R}^{B N *} \leq$ $\pi_{R}^{A N *} ; \pi_{R}^{C N *}<\pi_{R}^{A N *} ;$ when $0<\beta<\left(\left(1-\alpha-2 \alpha^{2}\right) / 2\right)$, $\pi_{R}^{C N *}<\pi_{R}^{B N *}$. When $\left(\left(1-\alpha-2 \alpha^{2}\right) / 2\right) \leq \beta<(1 / 2)$, $\pi_{R}^{C N *}>\pi_{R}^{B N *}$.

(iv) $\pi_{M}^{A N *}>\pi_{M}^{N N *} ; \pi_{M}^{B N *}>\pi_{M}^{N N *}$; when $([2(1+\alpha)-2(1+$ $2 \alpha)(1+\alpha) \sqrt{1+\alpha}] /[5+4 \alpha(2+\alpha)])<\beta<([2(1+\alpha)+$ $2(1+2 \alpha)(1+\alpha) \sqrt{1+\alpha}] /[5+4 \alpha(2+\alpha)]), \quad \pi_{M}^{C N *}>$ $\pi_{M}^{N N *}$, or else $\pi_{M}^{C N *} \leq \pi_{M}^{N N *} ;$ when $((2+6 \alpha-$ $2 \sqrt{1+\alpha}) /(5+9 \alpha))<\beta<((2+6 \alpha+2 \sqrt{1+\alpha}) /(5+9$ $\alpha)), \pi_{M}^{B N *}<\pi_{M}^{A N *}$, or else $\pi_{M}^{B N *} \geq \pi_{M}^{A N *} ;$ when $\sqrt{1+}$ $\alpha(2-3 \beta)(1+\alpha-\beta)-(1-\beta)[2+2 \alpha(1-\beta)-3 \beta]>$ $0, \pi_{M}^{C N *}>\pi_{M}^{A N *}$, or else $\pi_{M}^{C N *} \leq \pi_{M}^{A N *} ; \pi_{M}^{C N *}>\pi_{M}^{B N *}$.

Proposition 5 (i) shows the impact of fairness concerns on the profit margins of the retailer under no encroachment. The profit margin of the retailer in three fairness concern scenarios $(A, B, C)$ are lower than that when there is no fairness concern. That is, when the contract manufacturer does not encroach, the fairness concern decreases the profit margin of the retailer. When the advantageous inequity fairness concern parameter is sufficiently low, the profit margin of the retailer in scenario $B$ is 
lower than that of scenario $A$ and vice versa when the advantageous inequity fairness concern parameter is sufficiently high. Among four scenarios $(N, A, B, C)$, the profit margin of the retailer under scenario $C$ is the lowest. Proposition 5 (ii) shows the impact of fairness concerns on the wholesale price of the contract manufacturer under no encroachment. The fairness concern increases the wholesale price. When the advantageous inequity fairness concern parameter is sufficiently low, the wholesale price in scenario $B$ is higher than that of scenario $A$, and vice versa. Among four scenarios $(N, A, B, C)$, the wholesale price under scenario $C$ is the highest. Proposition 5 (iii) shows the impact of fairness concerns on the profit of the retailer under no encroachment. The fairness concern decreases the profit of the retailer. Proposition 5 (iv) shows the impact of fairness concerns on the profit of the contract manufacturer. For the contract manufacturer, the retailer's fairness concern and her own fairness concern both increase her profit.

\section{Proposition 6. Under encroachment,}

(i) $m^{A E *}>m^{N E *} ; m^{B E *}>m^{N E *} ; m^{C E *}>m^{N E *}$; when $(\alpha$ $/(5 \alpha+2))<\beta<(1 / 2), m^{B E *}>m^{A E *} ;$ when $0<\beta \leq(\alpha$ $/(5 \alpha+2)), \quad m^{B E *} \leq m^{A E *} ; \quad m^{C E *}>m^{A E *} ; \quad m^{C E *}>$ $m^{B E *}$.

(ii) $w^{A E *}<w^{N E *} ; w^{B E *}<w^{N E *} ; w^{C E *}<w^{N E *} ;$ when $(\alpha /$ $[4(1+2 \alpha)])<\beta<(1 / 2), w^{B E *}<w^{A E *} ;$ when $0<\beta \leq$ $(\alpha /[4(1+2 \alpha)]), w^{B E *} \geq w^{A E *} ; w^{C E *}<w^{A E *} ; w^{C E *}<$ $w^{B E *}$.

(iii) $\pi_{R}^{A E *}<\pi_{R}^{N E *} ; \pi_{R}^{B E *}>\pi_{R}^{N E *} ;$ when $\widehat{\beta}_{1}<\beta<\widehat{\beta}_{2}, \pi_{R}^{C E *}$ $>\pi_{R}^{N E *}$, or else $\pi_{R}^{C E *} \leq \pi_{R}^{N E *}$, where $\hat{\beta}_{1}=([1-$ $\left.\sqrt{(1+2 \alpha)^{2}(1+4 \alpha)(1+6 \alpha)}+2 \alpha(7+8 \alpha)\right] /[2+4 \alpha$ $(5+4 \alpha)]), \hat{\beta}_{2}=\left(\left[1+\sqrt{(1+2 \alpha)^{2}(1+4 \alpha)(1+6 \alpha)}\right.\right.$ $+2 \alpha(7+8 \alpha)] /[2+4 \alpha(5+4 \alpha)]) ; \quad$ when $\beta<\left(\left(7 \alpha+10 \alpha^{2}\right) / \quad\left(20 \alpha^{2}+11 \alpha-2\right)\right), \pi_{R}^{B E *}>\pi_{R}^{A E *}$, or else $\pi_{R}^{B E *} \leq \pi_{R}^{A E *} ; \pi_{R}^{C E *}>\pi_{R}^{A E *}$; when $0<\beta<\widehat{\beta}_{3}$, $\pi_{R}^{C E *}<\pi_{R}^{B E *} ;$ when $\widehat{\beta}_{3} \leq \beta<(1 / 2), \quad \pi_{R}^{C E *} \geq \pi_{R}^{B E *}$, where $\widehat{\beta}_{3}=([25+32 \alpha-\sqrt{121+48 \alpha(9+8 \alpha)}] /[4$ $(9+8 \alpha)])$.

(iv) $\pi_{M}^{A E *}<\pi_{M}^{N E *} ; \quad \pi_{M}^{B E *}<\pi_{M}^{N E *}, \quad \pi_{M}^{C E *}<\pi_{M}^{N E *} ;$ when $0<\beta<\left(\left(4 \alpha+5 \alpha^{2}\right) /\left[9(2+3 \alpha)^{2}\right]\right), \quad \pi_{M}^{B E *}>\pi_{M}^{A E *} ;$ when $\quad\left(\left(4 \alpha+5 \alpha^{2}\right) /\left[9(2+3 \alpha)^{2}\right]\right) \leq \beta<(1 / 2)$, $\pi_{M}^{B E *} \leq \pi_{M}^{A E *}$; when $(1-\beta)(2+3 \alpha)^{2} G-(9+26 \alpha+$ $\left.19 \alpha^{2}\right)[2+3 \alpha-2(1+\alpha) \beta]^{2}>0, U_{M}^{C E *}>\pi_{M}^{A E *}$, or else $U_{M}^{C E *} \leq \pi_{M}^{A E *}$, where $G=9+26 \alpha+19 \alpha^{2}-6(1+$ $\alpha)(3+4 \alpha) \beta+[9+8 \alpha(2+\alpha)] \beta^{2} ; \pi_{M}^{C E *}<\pi_{M}^{B E *}$.

Proposition 6 (i) shows that when the contract manufacturer encroaches, fairness concern will increase the retailer's profit margin. Among four scenarios $(A, B$, $C, N$ ), the profit margin of the retailer under scenario $C$ is the highest. Proposition 6 (ii) shows that the fairness concern decreases the wholesale price of the contract manufacturer. Among four scenarios $(A, B, C, N)$, the wholesale price of the contract manufacturer under scenario $C$ is the lowest. Proposition 6 (iii) shows that the retailer's fairness concern decreases his own profit; however, when the contract manufacturer has fairness concern, the profit of the retailer will be increased. Proposition 6 (iv) shows that the fairness concern decreases the contract manufacturer's profit.

In summary, Table 4 shows the effect of fairness concern on decisions and profits compared with scenario $N$ where there is no fairness concern.

Proposition 7. When the contract manufacturer encroaches, the retail prices of the private brand are the same, i.e., $p_{d}^{N E *}=p_{d}^{A E *}=p_{d}^{B E *}=p_{d}^{C E *}$.

Proposition 7 shows that the fairness concern has no impact on the retail price of the contract manufacturer's private brand.

Then, we investigate the impact of certain key parameters on the profits of both the manufacturer and the retailer. The default parameters are assumed as follows: $\{r=120, c=30, \alpha=0.1, \beta=0.2, t=20, d=0.3\}$. All parameters satisfy the conditions ensuring the existence of equilibriums. Several other groups of default parameters are tested as follows: $\{r=200, c=50, \alpha=0.2, \beta=0.3, t=$ $15, d=0.4\}, \quad\{r=500, c=100, \alpha=0.3, \beta=0.3, t=80, d=$ $0.6\}$, and $\quad\{r=1000, c=300, \alpha=0.2, \beta=0.1, t=100$, $d=0.2\}$. The results derived from the numerical examples are robust.

Figure 1(a) shows that under no encroachment, when there is no fairness concern of any member, the profit of the retailer is the highest. When the disadvantageous fairness concern parameter is sufficiently low and when both members have fairness concern, the profit of the retailer is the lowest. However, when the disadvantageous fairness concern parameter is sufficiently high and when the contract manufacturer has the fairness concern, the profit of the retailer is the lowest. Figure 1(b) shows that when both members have fairness concern, the profit of the contract manufacturer is the highest. When no one has fairness concern, the profit of the contract manufacturer is the lowest. Figure 1(c) illustrates that when both members have fairness concern, the profit of the whole supply chain is the highest. When disadvantageous fairness concern parameter is sufficiently low and when the contract manufacturer has fairness concern, the profit of the contract manufacturer is the lowest. However, when the disadvantageous fairness concern parameter is sufficiently high, the profit of the contract manufacturer is the lowest when no one has fairness concern. Under scenario $C$, the profit of the supply chain increases with the disadvantageous inequity parameter. The management insight here is that it is best for the whole supply chain that both members have fairness concern. The profit of the whole supply chain increases when they are more concerned about disadvantageous fairness.

Figure 2 has similar results with Figure 1. Besides, Figure 2 shows that when the advantageous inequity parameter increases, the profit of the retailer under scenario $A$ and scenario $C$ firstly decreases with advantageous inequity parameter and then increases with it. From Figures 1 and 2, 
TABLE 4: The impact of fairness concern on decisions and profits compared with scenario $N$.

\begin{tabular}{|c|c|c|c|c|}
\hline Decisions or profits & Scenario & $\begin{array}{c}\text { Retailer's fairness } \\
\text { concern } \\
(\text { scenario } A)\end{array}$ & $\begin{array}{c}\text { Contract manufacturer's } \\
\text { fairness } \\
\text { concern (scenario } B)\end{array}$ & $\begin{array}{c}\text { Both members' } \\
\text { fairness } \\
\text { concern (scenario } C \text { ) } \\
\end{array}$ \\
\hline \multirow[t]{2}{*}{ Profit margin } & $\begin{array}{c}\text { No } \\
\text { encroachment }\end{array}$ & - & 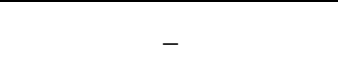 & - \\
\hline & Encroachment & + & + & + \\
\hline \multirow[t]{2}{*}{ Wholesale price } & $\begin{array}{c}\text { No } \\
\text { encroachment }\end{array}$ & + & + & + \\
\hline & Encroachment & - & - & - \\
\hline Private brand's retail price & Encroachment & No change & No change & No change \\
\hline \multirow[t]{2}{*}{ Retailer's profit/utility } & $\begin{array}{c}\text { No } \\
\text { encroachment }\end{array}$ & - & - & - \\
\hline & Encroachment & - & + & \pm \\
\hline \multirow{2}{*}{$\begin{array}{l}\text { Contract manufacturer's profit/ } \\
\text { utility }\end{array}$} & $\begin{array}{c}\text { No } \\
\text { encroachment }\end{array}$ & + & + & \pm \\
\hline & Encroachment & - & - & - \\
\hline
\end{tabular}

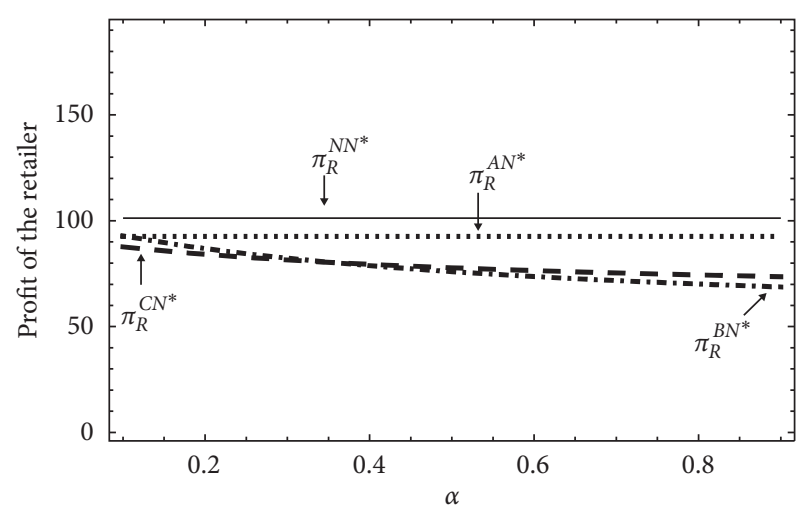

(a)

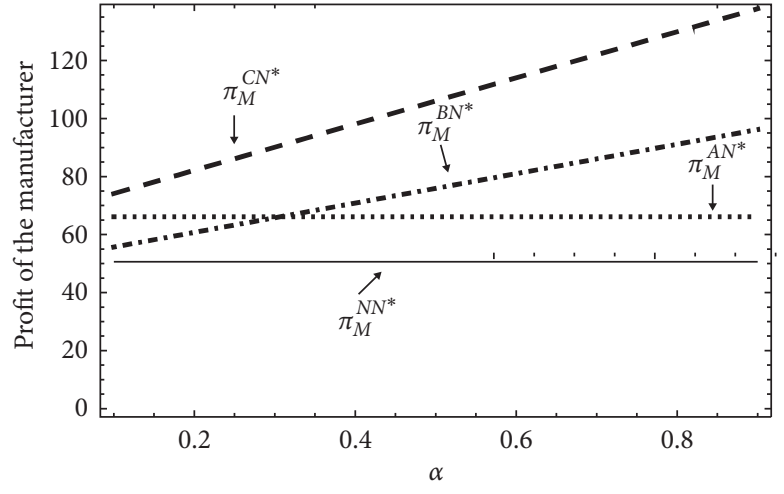

(b)

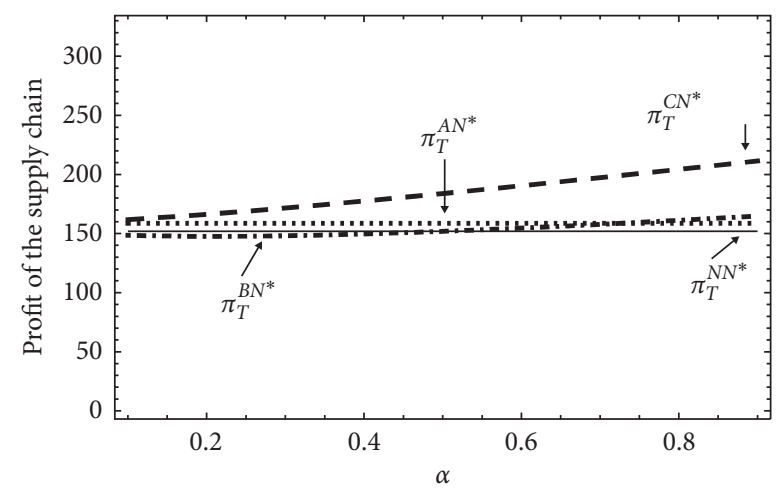

(c)

Figure 1: Profit of the players under four different scenarios $(A, B, C, N)$ versus $\alpha$ when the contract manufacturer does not encroach. (a) Profit of the retailer. (b) Profit of the contract manufacturer. (c) Profit of the whole supply chain.

it is best for the whole supply chain that both members have fairness concern. The profit of the whole supply chain increases when they are more concerned about fairness.

Figure 3(a) shows that the profit of the retailer is highest when only the contract manufacturer has fairness concern. When only the retailer has fairness concern, the profit of the retailer is the lowest. Under scenarios $A$ and $C$, the profit of the retailer decreases with the disadvantageous inequity parameter. Figure 3(b) presents that when no one has fairness concern, the contract manufacturer's profit is the highest and when both members have fairness concern, the profit of the contract manufacturer is the lowest. Figure 3(c) states that it is best for the supply chain that no one has fairness concern. When they are more concerned about disadvantageous inequity, the profit of the whole supply chain will decrease. The management insight here is that when the contract manufacturer has a private brand, it is best for the supply chain that no one has fairness concern.

Figure 4(a) shows that when the advantageous inequity concern parameter is sufficiently high and when both members 


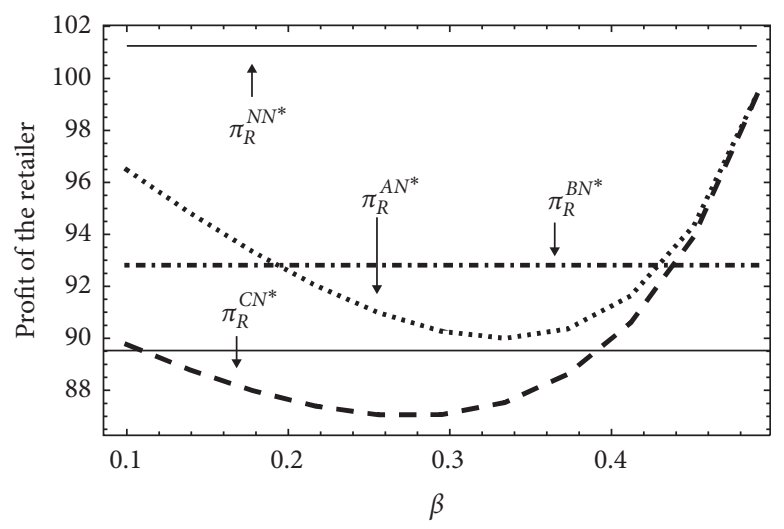

(a)

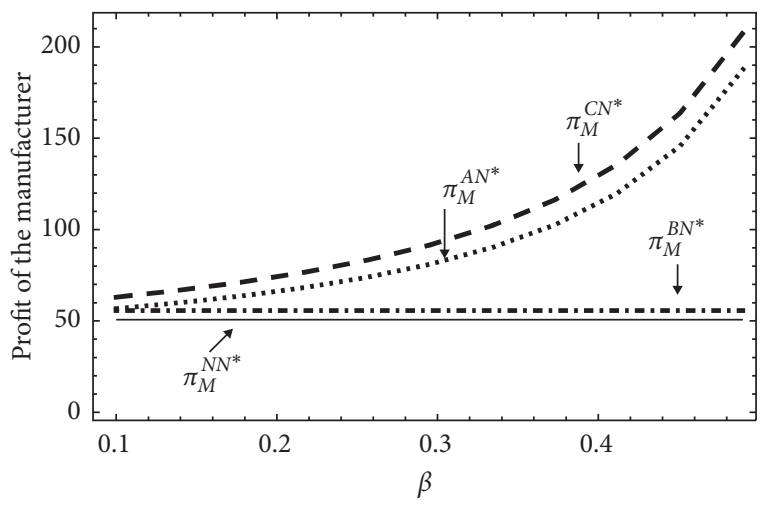

(b)

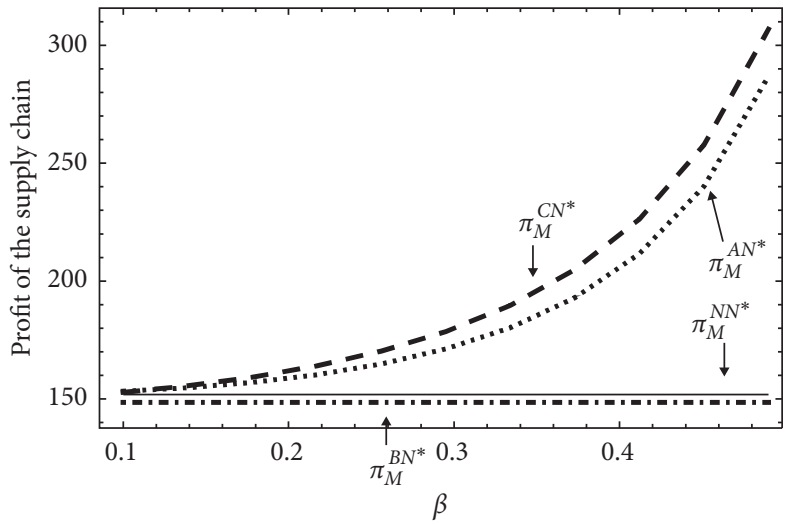

(c)

Figure 2: Profit of the players under four different scenarios $(A, B, C, N)$ versus $\beta$ when the contract manufacturer does not encroach. (a) Profit of the retailer. (b) Profit of the contract manufacturer. (c) Profit of the whole supply chain.

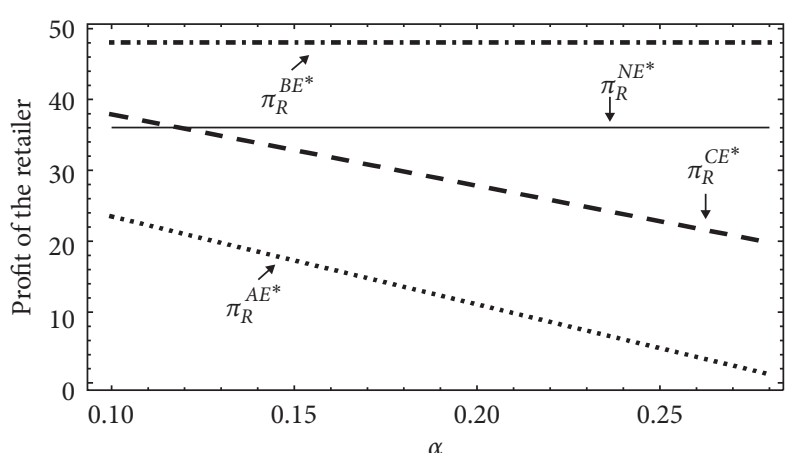

(a)

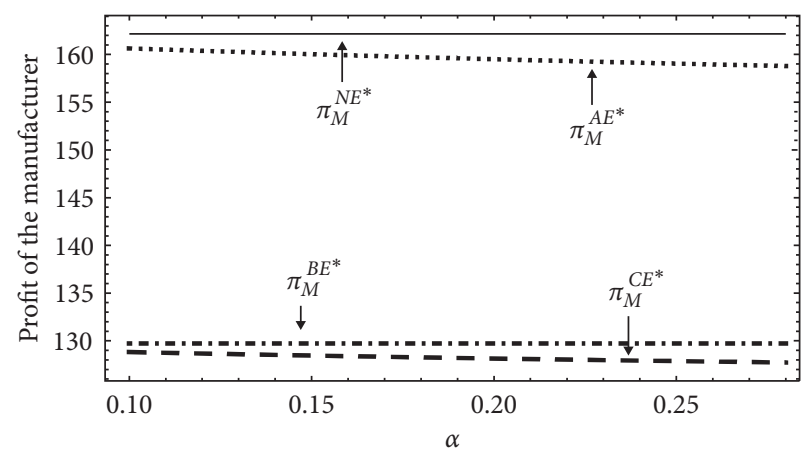

(b)

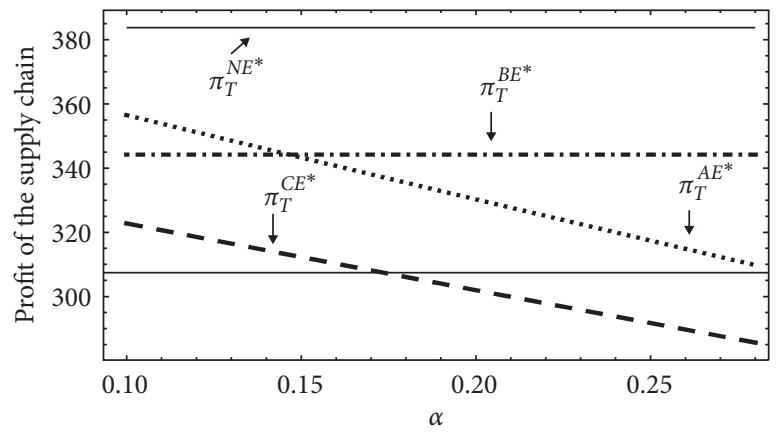

(c)

Figure 3: Profit of the players under four different scenarios $(A, B, C, N)$ versus $\alpha$ when the contract manufacturer encroaches. (a) Profit of the retailer. (b) Profit of the contract manufacturer. (c) Profit of the whole supply chain. 


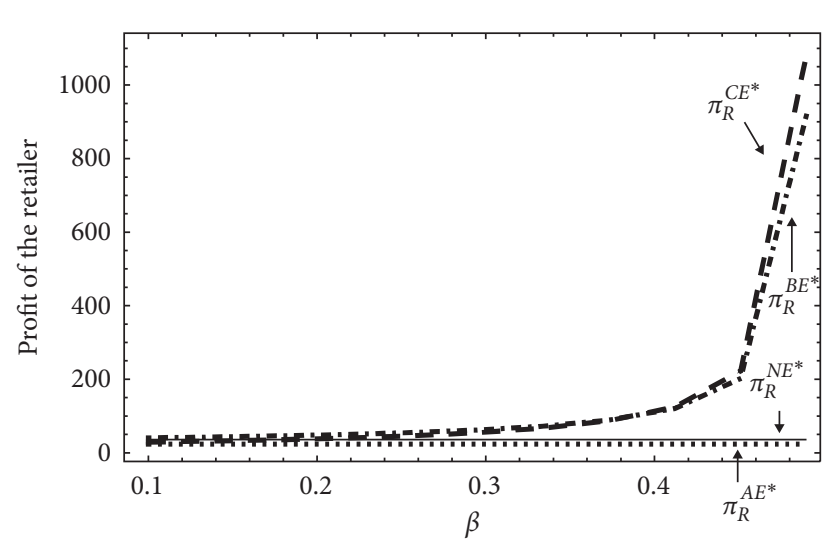

(a)

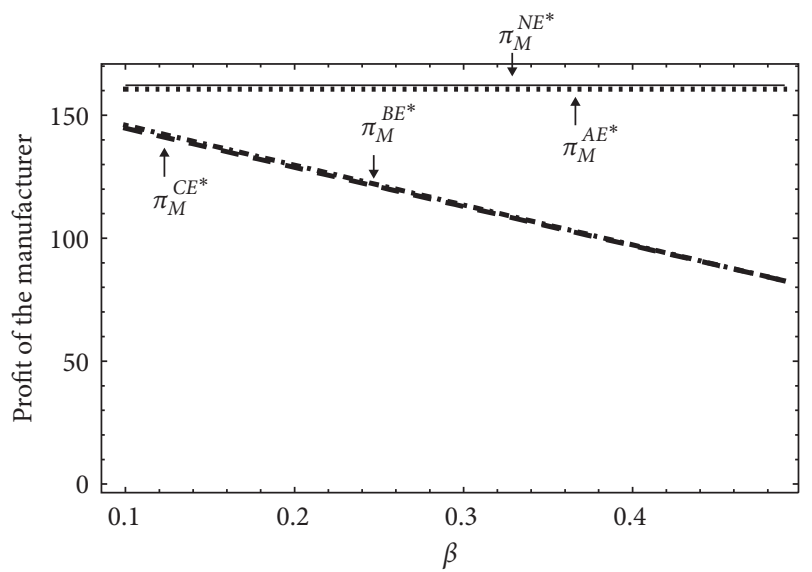

(b)

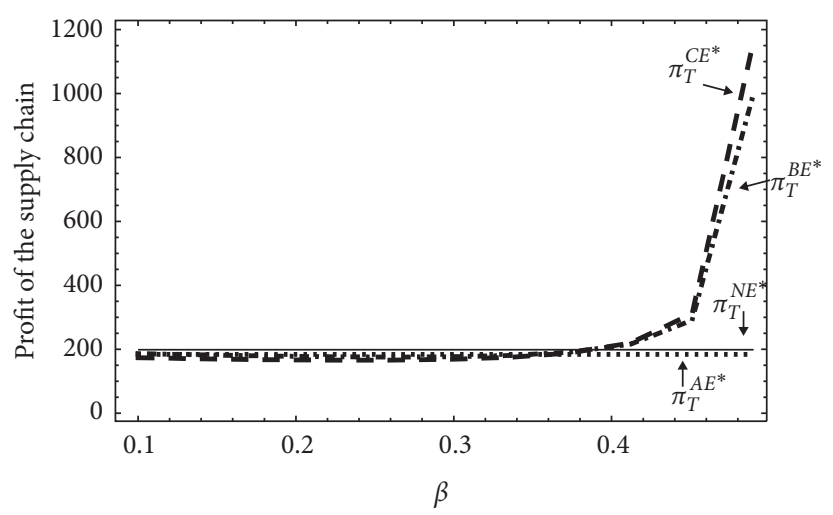

(c)

Figure 4: Profit of the players under four different scenarios $(A, B, C, N)$ versus $\beta$ when the contract manufacturer encroaches. (a) Profit of the retailer. (b) Profit of the contract manufacturer. (c) Profit of the whole supply chain.

have fairness concern, the profit of the retailer would be the highest. Figure 4(b) has similar results with Figure 3(b). Figure 4(c) shows that when the advantageous inequity concern parameter is sufficiently low, it is best for the whole supply chain that no one has fairness concern. However, when the advantageous inequity concern parameter is high enough, it is best for the whole supply chain that both members have fairness concern. Under scenarios $B$ and $C$, the profit of the whole supply chain increases with the advantageous inequity concern parameter when it is sufficiently high. The management insight here is that when the contract manufacturer has a private brand, it is best for the supply chain that no one has fairness concern when the advantageous inequity concern parameter is sufficiently low. However, when the advantageous inequity concern parameter is sufficiently high, it is best for the supply chain that both members have fairness concern.

\section{Conclusions}

This paper investigates a supply chain consisting of one contract manufacturer and one retailer who owns a wellknown brand selling in consumer market. The contract manufacturer provides production service for the retailer. The contract manufacturer's encroachment strategy and the impact of fairness concern on decisions and profits are investigated. The results show that when no member has fairness concern, the contract manufacturer always has motivation to encroach. However, when the retailer, the contract manufacturer, or both members have fairness concern, only if the reservation price is sufficiently high, the contract manufacturer will encroach. That is, the fairness concern has certain strength to stop the contract manufacturer to establish a private brand. When the reservation price is sufficiently low, the encroachment of the contract manufacturer benefits the retailer, or else it will harm the benefit of the retailer. Under encroachment strategy, the fairness concern decreases the profit margin of the retailer and increases wholesale price. However, under no encroachment strategy, the impact of fairness concern on pricing decisions is opposite except that the fairness concern has no impact on the retail price of the private brand. Under no encroachment strategy, the fairness concern has negative impact on the retailer's profit. However, under encroachment, the manufacturer's fairness concern can increase the retailer's profit. The fairness concern decreases the contract manufacturer's profit no matter what the form it is. Numerical examples show that under no encroachment, it is best for the whole supply chain that both members have fairness concern. The profit of the whole supply chain increases when they are more concerned about fairness. However, under encroachment, it is 
best for the supply chain that no one has fairness concern when the advantageous inequity concern parameter is sufficiently low. When the advantageous inequity concern parameter is sufficiently high, it is best for the supply chain that both members have fairness concern.

This study can be extended in the following aspects in the future research. Firstly, this paper derives demand function from the Hotelling model which indicates that the contract manufacturer's private brand has symmetric position with retailer's existing brand. However, consumers may have different perception for different brands in reality, especially for new brands. Thus, the demand function can be modelled in a different way. Secondly, this research assumes that supply chain members are completely information symmetric. It would be interesting to investigate how the information asymmetry affects the results. Thirdly, in big data era, real data can be used to make the model closer to the reality.

\section{Appendix}

\section{A. Proof of Proposition 1}

A.1. Equilibrium under Scenario NN. Because $\left(\mathrm{d}^{2} \pi_{M}^{N N} /\right.$ $\left.\mathrm{d} w^{2}\right)=-(4 / t)<0, \pi_{M}^{N N}$ is a concave function of $w$. By solving the first-order condition $\left(\mathrm{d} \pi_{M}^{N N} / \mathrm{d} w\right)=0$, we can get the reaction function of the contract manufacturer $w^{N N}(m)=((c-m+r) / 2)$. By Inserting $w^{N N}(m)$ into retailer's profit function, we can get $\pi_{R}^{N N}(m)$. Because $\left(\mathrm{d}^{2} \pi_{R}^{N N}(m) / \mathrm{d} m^{2}\right)=-(2 / t)<0, \quad \pi_{R}^{N N}(m)$ is a concave function of $m$. By solving the first-order condition $\left(\mathrm{d} \pi_{R}^{N N}(m) / \mathrm{d} m\right)=0$, we can get the optimal profit function of the retailer as $m^{N N *}=((r-c) / 2)$. Then, we can get the contract manufacturer's optimal wholesale price as $w^{N N *}=((r+3 c) / 4)$. Finally, we can get the profit of the retailer and the contract manufacturer as $\pi_{R}^{N N *}=$ $\left((r-c)^{2} /(4 t)\right)$ and $\pi_{M}^{N N *}=\left((r-c)^{2} /(8 t)\right)$, respectively.

A.2. Equilibrium under Scenario NE. Because $\left(\partial^{2} \pi_{M}^{N E} /\right.$ $\left.\partial w^{2}\right)=-(3 / t)<0$ and $\left(\partial^{2} \pi_{M}^{N E} / \partial w^{2}\right) \cdot\left(\partial^{2} \pi_{M}^{N E} / \partial p_{2}^{2}\right)-\left(\partial^{2}\right.$ $\left.\pi_{M}^{N E} / \partial w \partial p_{2}\right)^{2}=\left(8 / t^{2}\right)>0, \pi_{M}^{N E}$ is a concave function of $\left(w, p_{2}\right)$. By solving the first-order conditions $\left(\partial \pi_{M}^{N E} /\right.$ $\partial w)=0,\left(\partial \pi_{M}^{N E} / \partial p_{2}\right)=0$, we can get the reaction function of the contract manufacturer $w^{N E}(m)=((2 c-2 m+2 r+\mathrm{d} t) / 4)$ and $p_{2}^{N E}(m)=((2 c+2 r+\mathrm{d} t) / 4)$. By inserting $w^{N E}(m)$ and $p_{2}^{N E}(m)$ into the retailer's profit function, we can get $\pi_{R}^{N E}(m)$. Because $\left(\mathrm{d}^{2} \pi_{R}^{N E}(m) / \mathrm{d} m^{2}\right)=-(3 / 2 t)<0, \pi_{R}^{N E}(m)$ is a concave function of $m$. By solving the first-order condition $\left(\mathrm{d} \pi_{R}^{N E}(m) / \mathrm{d} m\right)=0$, we can get the optimal profit function of the existing brand as $m^{N E *}=((2 r+\mathrm{d} t-2 c) / 6)$. By inserting $m^{N E *}$ into $w^{N E}(m)$ and $p_{2}^{N E}(m)$, we can get the contract manufacturer's optimal decisions $w^{N E *}$ and $p_{2}^{N E *}$. The retailer's profit is $\pi_{R}^{N E *}=\left((2 r+\mathrm{d} t-2 c)^{2} /(48 t)\right)$. The contract manufacturer's profit is $\pi_{M}^{N E *}=\left(3(2 r+\mathrm{d} t-2 c)^{2} /(32 t)\right)$.

A.3. Equilibrium under Scenario AN. Because $\left(\partial^{2} \pi_{M}^{A N} /\right.$ $\left.\partial w^{2}\right)=-(4 / t)<0, \pi_{M}^{A N}$ is a concave function of $w$. By solving the first-order condition $\left(\mathrm{d} \pi_{M}^{A N} / \mathrm{d} m\right)=0$, we can get the reaction function of the contract manufacturer $w^{A N}(m)=$ $((c-m+r) / 2)$. By inserting $w^{A N}(m)$ into retailer's utility function, we can get $U_{R}^{A N}(m) . \quad\left(\partial^{2} U_{R}^{A N}(m) / \partial m^{2}\right)=$ $-(2 / t)+(3 \beta / t)$. Only if $\beta<(2 / 3), U_{R}^{A N}(m)$ is a concave function of $m$. By solving the first-order condition $\left(\mathrm{d} U_{R}^{A N}(m) / \partial m\right)=0$, we can get the optimal profit function of the retailer as $m^{A N *}=((r-c)(1-2 \beta) /(2-3 \beta))$. Then, we can get the contract manufacturer's optimal wholesale price as $w^{A N *}=((3 c+r-5 c \beta-r \beta) /(4-6 \beta))$. Finally, we can get the utility of the retailer as $U_{R}^{A N *}=\left((r-c)^{2}(1-\right.$ $\left.\beta)^{2} /[2 t(2-3 \beta)]\right)$. The profit of the contract manufacturer is $\pi_{M}^{A N *}=\left((r-c)^{2}(1-\beta)^{2} /\left[2 t(2-3 \beta)^{2}\right]\right)$. In order to ensure the positivity of the profit margin, $\beta<(1 / 2)$ must be satisfied. Thus, $\beta<(1 / 2)$ can ensure both concavity and positivity of the equilibrium.

A.4. Equilibrium under Scenario $A E$. Because $\left(\partial^{2} \pi_{M}^{A E} / \partial w^{2}\right)=$ $-(3 / t)<0$ and $\left(\partial^{2} \pi_{M}^{A E} / \partial w^{2}\right) \cdot\left(\partial^{2} \pi_{M}^{A E} / \partial p_{2}^{2}\right)-\left(\partial^{2} \pi_{M}^{A E} / \partial w\right.$ $\left.\partial p_{2}\right)^{2}=\left(8 / t^{2}\right)>0, \pi_{M}^{A E}$ is a concave function of $\left(w, p_{2}\right)$. By solving the first-order conditions $\left(\partial \pi_{M}^{A E} / \partial w\right)=0,\left(\partial \pi_{M}^{A E} /\right.$ $\left.\partial p_{2}\right)=0$, we can get the reaction function of the contract manufacturer $w^{A E}(m)=((2 c-2 m+2 r+\mathrm{d} t) / 4)$ and $p_{2}^{A E}$ $(m)=((2 c+2 r+\mathrm{d} t) / 4)$. By inserting $w^{A E}(m)$ and $p_{2}^{A E}(m)$ into the retailer's utility function, we can get $U_{R}^{A E}(m)$. Because $\left(\mathrm{d}^{2} U_{R}^{A E}(m) / \mathrm{d} m^{2}\right)=-(3 /(2 t))-(9 \alpha /(4 t))<0, \quad \pi_{R}^{N E}$ $(m)$ is a concave function of $m$. By solving the first-order condition $\left(\mathrm{d} \pi_{R}^{N E}(m) / \mathrm{d} m\right)=0$, we can get the optimal profit function of the existing brand as $m^{A E *}=((\mathrm{d} t+$ $2 r-2 c)(1+2 \alpha) /(6+9 \alpha))$. By inserting $m^{A E *}$ into $w^{A E}(m)$ and $p_{2}^{A E}(m)$, we can get the contract manufacturer's optimal decisions $w^{A E *}$ and $p_{2}^{A E *}$. The retailer's utility is $U_{R}^{A E *}=$ $\left((2 r+\mathrm{d} t-2 c)^{2}\left(1-2 \alpha-5 \alpha^{2}\right) /[24 t(2+3 \alpha)]\right)$. The contract manufacturer's profit is $\pi_{M}^{A E *}=\left((2 r+\mathrm{d} t-2 c)^{2}(9+\right.$ $\left.\left.26 \alpha+19 \alpha^{2}\right) /\left[24 t(2+3 \alpha)^{2}\right]\right)$. Note that $U_{R}^{A E *}$ must be nonnegative, or else the retailer will abandon the supply chain. That is, $0<\alpha \leq((\sqrt{6}-1) / 5)$.

A.5. Equilibrium under Scenario BN. Because $\left(\partial^{2} \pi_{M}^{B N} /\right.$ $\left.\partial w^{2}\right)=-(4 / t)-(4 \alpha / t)<0, \pi_{M}^{B N}$ is a concave function of $w$. By solving the first-order condition $\left(\mathrm{d} \pi_{M}^{B N} / \mathrm{d} w\right)=0$, we can get the reaction function of the contract manufacturer $w^{B N}(m)=((c-m+r+c \alpha+r \alpha) /[2(1+\alpha)])$. By inserting $w^{B N}(m)$ into retailer's profit function, we can get $\pi_{R}^{B N}(m)$. Because $\quad\left(\mathrm{d}^{2} \pi_{R}^{B N}(m) / \mathrm{d} m^{2}\right)=-((2+4 \alpha) /(t+t \alpha))<0$, $\pi_{R}^{B N}(m)$ is a concave function of $m$. By solving the first-order condition $\left(\mathrm{d} \pi_{R}^{B N}(m) / \partial m\right)=0$, we can get the optimal profit function of the retailer as $m^{B N *}=((r-c)(1+\alpha) /(2+4 \alpha))$. Then, we can get the contract manufacturer's optimal wholesale price as $w^{B N *}=((3 c+r+4 c \alpha+4 r \alpha) /(4+8 \alpha))$. Also, we can get the utility of the contract manufacturer as $U_{M}^{B N *}=\left((r-c)^{2}(1+\alpha) /(8 t)\right)$. The profit of the retailer is $\pi_{R}^{B N *}=\left((r-c)^{2}(1+\alpha) /[4(t+2 t \alpha)]\right)$.

A.6. Equilibrium under Scenario $B E$. Because $\left(\partial^{2} U_{M}^{B E} / \partial w^{2}\right)=$ $-(3(1-\beta) / t)<0$ and $\left(\partial^{2} \pi_{M}^{B E} / \partial w^{2}\right) \cdot\left(\partial^{2} \pi_{M}^{B E} / \partial p_{2}^{2}\right)-\left(\partial^{2} \pi_{M}^{B E}\right.$ $\left./ \partial w \partial p_{2}\right)^{2}=\left(8(1-\beta)^{2} / t^{2}\right)>0, U_{M}^{B E}$ is a concave function of $\left(w, p_{2}\right)$. By solving the first-order conditions $\left(\partial U_{M}^{B E} /\right.$ $\partial w)=0,\left(\partial U_{M}^{B E} / \partial p_{2}\right)=0$, we can get the reaction function of 
the contract manufacturer $w^{B E}(m)=([2 c+2 r+$ $\mathrm{d} t-2 m /(1-\beta)] / 4)$ and $p_{2}^{B E}(m)=([2(c+r)+\mathrm{d} t] / 4)$. By inserting $w^{B E}(m)$ and $p_{2}^{B E}(m)$ into the retailer's utility function, we can get $\pi_{R}^{B E}(m) . \quad\left(\partial^{2} \pi_{R}^{B E}(m) / \mathrm{d} m^{2}\right)=$ $-(3\{1-1 /[2(1-\beta)]\} / t)$. Only if $\beta<(1 / 2), \pi_{R}^{B E}(m)$ is a concave function of $m$. By solving the first-order condition $\left(\mathrm{d} \pi_{R}^{B E}(m) / \mathrm{d} m\right)=0$, we can get the optimal profit function of the existing brand as $m^{B E *}=((\mathrm{d} t+2 r-2 c)(1-\beta) /(6-12 \beta))$. By inserting $m^{B E *}$ into $w^{B E}(m)$ and $p_{2}^{B E}(m)$, we can get the contract manufacturer's optimal decisions $w^{B E *}=((2 r+$ $\mathrm{d} t)(1-3 \beta)+c(4-6 \beta) /(6-12 \beta))$ and $p_{2}^{B E *}=([2(c+r)+$ $\mathrm{d} t] / 4)$. The retailer's profit is $\pi_{R}^{B E *}=\left((2 r+\mathrm{d} t-2 c)^{2}(1-\right.$ $\beta) /[48 t(1-2 \beta)])$. The contract manufacturer's utility is $U_{M}^{B E *}=\left(3(2 r+\mathrm{d} t-2 c)^{2}(1-\beta) /(32 t)\right)$.

A.7. Equilibrium under Scenario $C N$. Because $\left(\partial^{2} U_{M}^{C N} /\right.$ $\left.\partial w^{2}\right)=-(4 / t)-(4 \alpha / t)<0, U_{M}^{C N}$ is a concave function of $w$. By solving the first-order condition $\left(\mathrm{d} U_{M}^{C N} / \mathrm{d} w\right)=0$, we can get the reaction function of the contract manufacturer as $w^{C N}(m)=((c-m+r+c \alpha+r \alpha) /[2(1+\alpha)])$. By inserting $w^{C N}(m)$ into retailer's profit function, we can get $\pi_{R}^{C N}(m)$. Because $\mathrm{d}^{2} \pi_{R}^{C N}(m) / \mathrm{d} m^{2}=-((1+2 \alpha)[2+2 \alpha(1-\beta)-3 \beta]$ $\left./\left[t(1+\alpha)^{2}\right]\right)$, when $2+2 \alpha(1-\beta)-3 \beta>0$, i.e., $\beta<((2 \alpha+$ $2) /(2 \alpha+3)), \pi_{R}^{C N}(m)$ is a concave function of $m$. By solving the first-order condition $\left(\mathrm{d} \pi_{R}^{C N}(m) / \partial m\right)=0$, we can get the optimal profit function of the retailer as $m^{C N *}=((r-c)$ $\left.(1+\alpha)^{2}(1-2 \beta) /\{(1+2 \alpha)[2+2 \alpha(1-\beta)-3 \beta]\}\right)$. Then, we can get the contract manufacturer's optimal wholesale price as $w^{C N *}=\left(\left(c+r+c \alpha+r \alpha-m^{C N *}\right) /[2(1+\alpha)]\right)$. Also, we can get the utility of the contract manufacturer as $U_{M}^{B N *}=$ $\left((r-c)^{2} \quad(1+\alpha)(1+\alpha-\beta)^{2} / \quad\left\{2 t[2+2 \alpha(1-\beta)-3 \beta]^{2}\right\}\right)$. The profit of the retailer is $\pi_{R}^{B N *}=\left((r-c)^{2}(1+\right.$ $\left.\alpha-\beta)^{2} /\{2 t(1+2 \alpha)[2+2 \alpha(1-\beta)-3 \beta]\}\right)$. In order to ensure the positivity of the profit margin, $\beta<(1 / 2)$ must be satisfied. Because $((2 \alpha+2) /(2 \alpha+3))-(1 / 2)=((2 \alpha+1) /[2(2 \alpha+3)])>0$, $\beta<(1 / 2)$ can ensure both concavity and positivity of the equilibrium.

A.8. Equilibrium under Scenario CE. Because $\left(\partial^{2} U_{M}^{C E} /\right.$ $\left.\partial w^{2}\right)=-(3(1-\beta) / t)<0$ and $\left(\partial^{2} U_{M}^{C E} / \quad \partial w^{2}\right) \cdot\left(\partial^{2} \quad U_{M}^{C E} / \partial\right.$ $\left.p_{2}^{2}\right)-\left(\partial^{2} U_{M}^{C E} / \partial w \partial p_{2}\right)^{2}=\left(8(1-\beta)^{2} / t^{2}\right)>0, U_{M}^{C E}$ is a concave function of $\left(w, p_{2}\right)$. By solving the first-order conditions $\left(\partial U_{M}^{C E} / \partial w\right)=0,\left(\partial U_{M}^{C E} / \partial p_{2}\right)=0$, we can get the reaction function of the contract manufacturer as $w^{C E}(m)=$ $([2 c+2 r+\mathrm{d} t-2 m /(1-\beta)] / 4) \quad$ and $\quad p_{2}^{C E}(m)=$ $([2(c+r)+\mathrm{d} t] / 4)$. By inserting $w^{C E}(m)$ and $p_{2}^{C E}(m)$ into the retailer's utility function, we can get $\pi_{R}^{C E}(m) \cdot \mathrm{d}^{2} U_{R}^{C E}$ $(m) / \mathrm{d} m^{2}=-\left(3(1-2 \beta)[2+3 \alpha-2(1+\alpha) \beta] /\left[4 t(1-\beta)^{2}\right]\right)$. Let $f(\beta)=3(1-2 \beta)[2+3 \alpha-2(1+\alpha) \beta]$. Because $\left(\mathrm{d}^{2} f(\beta) /\right.$ $\left.\mathrm{d} \beta^{2}\right)=24(1+\alpha)>0, f(\beta)$ is a convex function of $\beta$. Let $f(\beta)=0$, and we can get two roots $\beta_{1}=1 / 2$ and $\beta_{2}=((2+3 \alpha) /[2(1+\alpha)])$. Since $\beta_{2}=((2+3 \alpha) /[2(1+\alpha)])>1$, we can omit this root. Thus, when $\beta<(1 / 2), f(\beta)>0$. Then, $U_{R}^{C E}(m)$ is a concave function of $m$. By solving the first-order condition $\left(\mathrm{d} U_{R}^{C E}(m) / \mathrm{d} m\right)=0$, we can get the optimal profit function of the existing brand as $m^{C E *}=((\mathrm{d} t+2 r-$ $\left.2 c)(1+2 \alpha)(1-\beta)^{2} /\{3(1-2 \beta)[2+3 \alpha-2(1+\alpha) \beta]\}\right)$.

By inserting $m^{C E *}$ into $w^{C E}(m)$ and $p_{2}^{C E}(m)$, we can get the contract manufacturer's optimal decisions as $w^{C E *}=$ $w^{C E}(m)=\left(\left[2 c+2 r+\mathrm{d} t-2 m^{C E *} /(1-\beta)\right] / 4\right)$ and $p_{2}^{C E *}=$ $([2(c+r)+d t] / 4)$. Then, we can get the retailer and the contract manufacturer's utility.

\section{B. Proof of Proposition 3}

B.1. The Encroachment Strategy of Scenario N. From Propositions 1, the profit of the contract manufacturer under two different encroachment strategies is $\pi_{M}^{N N *}=\left((r-c)^{2} /(8 t)\right)$ and $\pi_{M}^{N E *}=\left(3(2 r+\mathrm{d} t-2 c)^{2} /(32 t)\right)$, respectively. $\pi_{M}^{N E *}-$ $\pi_{M}^{N N *}=\left(\left[3(2 r+\mathrm{d} t-2 c)^{2}-4(r-c)^{2}\right] /(32 t)\right)$. Only when $3(2 r+d t-2 c)^{2}-4(r-c)^{2} \geq 0$, the contract manufacturer will establish her private brand. That is, $r \geq c-((3+$ $\sqrt{3}) \mathrm{d} t / 4)$. Because we assume that the reservation price is always higher than the cost $(r>c), r \geq c-((3+\sqrt{3}) \mathrm{d} t / 4)$ is always satisfied.

B.2. The Encroachment Strategy of Scenario A. From Propositions 1, the profit of the contract manufacturer under two different encroachment strategies is $\pi_{M}^{A N *}=\left((r-c)^{2}(1-\right.$ $\left.\beta)^{2} /\left[2 t(2-3 \beta)^{2}\right]\right)$ and $\pi_{M}^{A E *}=\left((2 r+\mathrm{d} t-2 c)^{2}(9+26 \alpha+19\right.$ $\left.\left.\alpha^{2}\right) /\left[24 t(2+3 \alpha)^{2}\right]\right)$, respectively. $\pi_{M}^{A E *}-\pi_{M}^{A N *}=((2 r+\mathrm{d} t-$ $\left.2 c)^{2}\left(9+26 \alpha+19 \alpha^{2}\right) /\left[24 t(2+3 \alpha)^{2}\right]\right)-\left((r-c)^{2}(1-\beta)^{2} / \quad[2 t\right.$ $\left.\left.(2-3 \beta)^{2}\right]\right)$. Only when $M=(2 r+\mathrm{d} t-2 c)^{2}\left(9+26 \alpha+19 \alpha^{2}\right)$ $(2-3 \beta)^{2}-12(r-c)^{2}(1-\beta)^{2} \quad(2+3 \alpha)^{2} \geq 0$, the contract manufacturer will establish her private brand. That is, $r \geq([2 c L-2 \sqrt{3} c(2+3 \alpha)(1-\beta)-\mathrm{d} t L] /[2 L-2 \sqrt{3}(2+3 \alpha)(1$ $-\beta)])$, where $L=\sqrt{9+\alpha(26+19 \alpha)}(2-3 \beta)$.

B.3. The Encroachment Strategy of Scenario B. From Propositions 1, the utility of the contract manufacturer under two different encroachment strategies is $U_{M}^{B N *}=\left((r-c)^{2}(1+\right.$ $\alpha) /(8 t))$ and $U_{M}^{B E *}=\left(3(2 r+\mathrm{d} t-2 c)^{2}(1-\beta) /(32 t)\right)$, respectively. $\quad U_{M}^{B E *}-U_{M}^{B N *}=\left(3(2 r+\mathrm{d} t-2 c)^{2}(1-\beta)-4(r-c)^{2}\right.$ $(1+\alpha) /(32 t))$. Only when $N=3(2 r+\mathrm{d} t-2 c)^{2}(1-\beta)-4(r-$ $c)^{2}(1+\alpha) \geq 0$, the contract manufacturer will establish her private brand. That is, $r \geq(\{2 c(2+\alpha-3 \beta)-\mathrm{d} t[3+$ $\sqrt{3(1-\alpha)} \sqrt{1-\beta}-3 \beta]\} /[2(2+\alpha-3 \beta)])$.

B.4. The Encroachment Strategy of Scenario C. From Propositions 1, the utility of the contract manufacturer under two different encroachment strategies is $U_{M}^{C N *}=\left((r-c)^{2}(1+\alpha)\right.$ $\left.(1+\alpha-\beta)^{2} /\left\{2 t[2+2 \alpha(1-\beta)-3 \beta]^{2}\right\}\right)$ and $U_{M}^{C E *}=((2 r+\mathrm{d} t-$ $\left.2 c)^{2}(1-\beta) G /\left\{24 t[2+3 \alpha-\quad 2(1+\alpha) \beta]^{2}\right\}\right), \quad$ respectively, where $G=9+26 \alpha+19 \alpha^{2}-6(1+\alpha)(3+4 \alpha) \beta+[9+8 \alpha(2$ $+\alpha)] \beta^{2} \cdot U_{M}^{C E *}-U_{M}^{C N *}=\left((2 r+\mathrm{d} t-2 c)^{2}(1-\beta) G /\{24 t[2+3 \alpha-\right.$ $\left.\left.2(1+\alpha) \beta]^{2}\right\}\right)-\left((r-c)^{2}(1+\alpha)(1+\alpha-\beta)^{2} /\{2 t[2+2 \alpha(1-\beta)-\right.$ $\left.\left.3 \beta]^{2}\right\}\right)$. Only when $U_{M}^{C E *}-U_{M}^{C N *} \geq 0$, the contract manufacturer will establish her private brand. That is, $M \geq 0$, where $M=(2 r+\mathrm{d} t-2 c)^{2} \quad(1-\beta)[2+2 \alpha(1-\beta)-3 \beta]^{2} G-12(r-c)^{2}$ $(1+\alpha)(1+\alpha-\beta)^{2}[2+3 \alpha-2(1+\alpha) \beta]^{2}, \quad G=9+\quad 26 \alpha+19 \alpha^{2}$ $-6(1+\alpha)(3+4 \alpha) \beta+[9+8 \alpha(2+\alpha)] \beta^{2}$. 
That is, $r \geq((2 c J-\mathrm{d} t J-c K) /(2 J-K))$, where $J=[2+$ $2 \alpha(1-\beta)-3 \beta] \sqrt{1-\beta} \sqrt{G} \quad$ and $\quad K=2 \sqrt{3} \sqrt{1+\alpha}$ $(1+\alpha-\beta)[2+3 \alpha-2(1+\alpha) \beta]$.

\section{Proof of Proposition 4}

(i) $\pi_{R}^{N N *} / \pi_{R}^{N E *}=\left(2 \sqrt{3}(r-c) /(2 r+\mathrm{d} t-2 c)^{2}\right)$; when $r<(c+\mathrm{d} t /(2 \sqrt{3}-2)), \pi_{R}^{N N *}<\pi_{R}^{N E *}$, or else $\pi_{R}^{N N *}$ $\geq \pi_{R}^{N E *}$.

(ii) $\pi_{R}^{A N *}-\pi_{R}^{A E *}=\left((r-c)^{2}(1-\beta)^{2} \quad /[2 t(2-3 \beta)]\right)-$ $\left((2 r+\mathrm{d} t-2 c)^{2}\left(1-2 \alpha-5 \alpha^{2}\right) /[24 t(2+3 \alpha)]\right)$; when $r>((\mathrm{d} t-2 c) N /(1-2 N)), \quad \pi_{R}^{A N *}>\pi_{R}^{A E *}, \quad$ or else $\pi_{R}^{A N *} \leq \pi_{R}^{A E *}, \quad$ where $\quad N=$ $\sqrt{\left(\left(1-2 \alpha-5 \alpha^{2}\right)(2-3 \beta) /\left[12(1-\beta)^{2}(2+3 \alpha)\right]\right)}$.

(iii) $\pi_{R}^{B N *}-\pi_{R}^{B E *}=\left((r-c)^{2}(1+\alpha) /[4 t(1+2 \alpha)]\right)-((2 r$ $\left.+\mathrm{d} t-2 c)^{2}(1-\beta) /[48 t(1-2 \beta)]\right), \quad r>c+(\mathrm{d} t /(1-2 V))$, $\pi_{R}^{B N *}>\pi_{R}^{B E *}, \quad$ or else $\pi_{R}^{B N *} \leq \pi_{R}^{B E *}$, where $V=\sqrt{((1-\beta)(1+2 \alpha) /[14(1+\alpha)(1-2 \beta)])}$. (iv) $\pi_{R}^{C N *}-\pi_{R}^{C E *}=\left((r-c)^{2}(1+\alpha-\beta)^{2} /\{2 t(1+2 \alpha)\right.$ $[2+2 \alpha(1-\beta)-3 \beta]\})-\left((2 r+\mathrm{d} t-2 c)^{2} H /\{24 t(1-\right.$ $2 \beta)[2+3 \alpha-2(1+\alpha) \beta]\})$; when $r>c+(\mathrm{d} t /(1-2 Z))$, $\pi_{R}^{B N *}>\pi_{R}^{B E *}$, or else, $\pi_{R}^{C N *} \leq \pi_{R}^{C E *}$, where $Z=$ $\sqrt{\left(H(1+2 \alpha)[2+2 \alpha(1-\beta)-3 \beta] /\left\{12(1+\alpha-\beta)^{2}\right.\right.}$ $(1-2 \beta)[2+3 \alpha-2(1+\alpha) \beta]\})$.

\section{Proof of Proposition 5}

$$
\frac{m^{A N *}}{m^{N N *}}=\frac{(r-c)(1-2 \beta)}{2-3 \beta} \cdot \frac{2}{r-c}=\frac{2-4 \beta}{2-3 \beta}<1,
$$

that is, $m^{A N *}<m^{N N *}$.

$$
\frac{m^{B N *}}{m^{N N *}}=\frac{(r-c)(1+\alpha)}{2+4 \alpha} \cdot \frac{2}{r-c}=\frac{2+2 \alpha}{2+4 \alpha}<1,
$$

that is, $m^{B N *}<m^{N N *}$.

$$
m^{C N *}-m^{N N *}=\frac{(r-c)(1+\alpha)^{2}(1-2 \beta)}{(1+2 \alpha)[2+2 \alpha(1-\beta)-3 \beta]}-\frac{r-c}{2}=\frac{2(r-c)(1+\alpha)^{2}(1-2 \beta)-(r-c)(1+2 \alpha)[2+2 \alpha(1-\beta)-3 \beta]}{2(1+2 \alpha)[2+2 \alpha(1-\beta)-3 \beta]} .
$$

Since $\beta<((2 \alpha+2) /(2 \alpha+3)), \quad 2+2 \alpha(1-\beta)-3 \beta>0$. Let $g_{1}(\beta)=2(r-c) \quad(1+\alpha)^{2}(1-2 \beta)-(r-c)(1+2 \alpha)$ $[2+2 \alpha(1-\beta)-3 \beta] .\left(\mathrm{d} g_{1}(\beta) / \mathrm{d} \beta\right)=c-r<0$. That is, $g_{1}(\beta)$ is a decreasing function of $\beta$. Let $g_{1}(\beta)=0$, and we can get a root as $\left\{-2\left(\alpha+\alpha^{2}\right)\right\}$. When $\beta>-2\left(\alpha+\alpha^{2}\right), g_{1}(\beta)<0$. We know that $\beta>0$; thus, $g_{1}(\beta)<0$. Then, we can get $m^{C N *}-m^{N N *}<0$. That is, $m^{C N *}<m^{N N *}$.

$$
m^{B N *}-m^{A N *}=\frac{(r-c)(1+\alpha)}{2+4 \alpha}-\frac{(r-c)(1-2 \beta)}{2-3 \beta}=\frac{(r-c)(1+\alpha)(2-3 \beta)-(r-c)(1-2 \beta)(2+4 \alpha)}{(2+4 \alpha)(2-3 \beta)} .
$$

Because $\beta<(2 / 3), \quad 2-3 \beta>0 . \quad \operatorname{Let}_{2}(\beta)=(r-c)(1+$ $\alpha)(2-3 \beta)-(r-c)(1-2 \beta)(2+4 \alpha) . \quad g_{2}(\beta)$ is a linear function of $\beta .\left(\mathrm{d} g_{2}(\beta) / \mathrm{d} \beta\right)=(r-c)(1+5 \alpha)>0$. That is, $g_{2}(\beta)$ is an increasing function of $\beta$. Let $g_{2}(\beta)=0$, and we can get a root as $\{(2 \alpha /(1+5 \alpha))\}$. $\quad(2 \alpha /(1+$
$5 \alpha))-(1 / 2)=((-1-\alpha) /[2(1+5 \alpha)])<0$; thus, $\quad(2 \alpha /(1+$ $5 \alpha))<(1 / 2)$. When $(2 \alpha /(1+5 \alpha))<\beta<(1 / 2), \quad g_{2}(\beta)>0$, $m^{B N *}>m^{A N *}$. When $0<\beta \leq(2 \alpha /(1+5 \alpha)), \quad g_{2}(\beta) \leq 0$, $m^{B N *} \leq m^{A N *}$.

$$
\begin{aligned}
m^{C N *}-m^{A N *} & =\frac{(r-c)(1+\alpha)^{2}(1-2 \beta)}{(1+2 \alpha)[2+2 \alpha(1-\beta)-3 \beta]}-\frac{(r-c)(1-2 \beta)}{2-3 \beta} \\
& =\frac{(r-c)(1+\alpha)^{2}(1-2 \beta)(2-3 \beta)-(r-c)(1-2 \beta)(1+2 \alpha)[2+2 \alpha(1-\beta)-3 \beta]}{(1+2 \alpha)[2+2 \alpha(1-\beta)-3 \beta](2-3 \beta)} .
\end{aligned}
$$

From $\beta<(2 / 3)$ and $\beta<((2 \alpha+2) /(2 \alpha+3))$, we know that $(1+2 \alpha)[2+2 \alpha(1-\beta)-3 \beta](2-3 \beta)>0$. Let $g_{3}(\beta)=$ $(r-c)(1+\alpha)^{2}(1-2 \beta)(2-3 \beta)-(r-c)(1-2 \beta)(1+2 \alpha)$

$[2+2 \alpha(1-\beta)-3 \beta]$. Because $\quad\left(\partial^{2} g_{3}(\beta) / \partial \beta^{2}\right)=$ 
$4(c-r) \alpha(2+\alpha)<0, g_{3}(\beta)$ is a concave function of $\beta$. By solving $g_{3}(\beta)=0$, we can get two roots
$\{(1 / 2),(2(1+\alpha) /(2+\alpha))\}, \quad$ where $\quad(2(1+\alpha) /(2+\alpha))>1$. When $\beta<(1 / 2), g_{3}(\beta)<0$ and $m^{C N *} \leq m^{A N *}$.

$$
\begin{aligned}
m^{C N *}-m^{B N *} & =\frac{(r-c)(1+\alpha)^{2}(1-2 \beta)}{(1+2 \alpha)[2+2 \alpha(1-\beta)-3 \beta]}-\frac{(r-c)(1+\alpha)}{2+4 \alpha} \\
& =\frac{(r-c)(1+\alpha)^{2}(1-2 \beta)(2+4 \alpha)-(r-c)(1+\alpha)(1+2 \alpha)[2+2 \alpha(1-\beta)-3 \beta]}{(1+2 \alpha)[2+2 \alpha(1-\beta)-3 \beta](2+4 \alpha)} .
\end{aligned}
$$

Let $g_{4}(\beta)=(r-c) \quad(1+\alpha)^{2}(1-2 \beta)(2+4 \alpha)-(r-$ c) $(1+\alpha)(1+2 \alpha)[2+2 \alpha(1-\beta)-3 \beta]$. We can get the unique root as $\{0\}$. Because $\left(\mathrm{d} g_{4}(\beta) / \mathrm{d} \beta\right)=-(r-$ c) $(1+\alpha)(1+2 \alpha)^{2}<0, g_{4}(\beta)$ is a decreasing function of $\beta$. Thus, when $\beta>0, g_{4}(\beta)<0$, that is, $m^{C N *}<m^{B N *}$.

$$
w^{A N *}-w^{N N *}=\frac{3 c+r-5 c \beta-r \beta}{2(2-3 \beta)}-\frac{r+3 c}{4}=\frac{(r-c) \beta}{4(2-3 \beta)} .
$$

Since $\beta<(2 / 3), 2-3 \beta>0$, and the reservation price of the consumer is higher than the cost, i.e., $r-c>0$. As a result, $w^{A N *}>w^{N N *} \cdot w^{B N *}-w^{N N *}=(2(r-c) \alpha /[4(1+2 \alpha)])>0$. As a result, $\quad w^{B N *}>w^{N N *}$. $\quad w^{C N *}-w^{N N *}=((r-c)[4 \alpha$ $(1+\alpha)(1-\beta)+\beta] /\{4(1+2 \alpha)[2+2 \alpha(1-\beta)-3 \beta]\})>0$, $w^{C N *}>w^{N N *}$.

$$
w^{B N *}-w^{A N *}=\frac{3 c+r+4 c \alpha+4 r \alpha}{4+8 \alpha}-\frac{3 c+r-5 c \beta-r \beta}{4-6 \beta} .
$$

Solving $w^{B N *}-w^{A N *}=0$, we can get a unique root as $[4 \alpha /(1+8 \alpha)] . \quad \partial\left(w^{B N *}-w^{A N *}\right) / \partial \beta=-((r-c) /$ $\left.\left[2(2-3 \beta)^{2}\right]\right)<0,\left(w^{B N *}-w^{A N *}\right)$ is a decreasing function of $\beta$. Because $(4 \alpha /(1+8 \alpha))-(1 / 2)=-(1 /[2(1+8 \alpha)])<0$, $(4 \alpha /(1+8 \alpha))<(1 / 2)$. When $0<\beta<(4 \alpha /(1+8 \alpha))$, $w^{B N *}>w^{A N *}$. When $(4 \alpha /(1+8 \alpha)) \leq \beta<(1 / 2), w^{B N *} \leq w^{A N *}$.

$$
w^{C N *}-w^{A N *}=\frac{(r-c) \alpha(1-2 \beta)[4+4 \alpha(1-\beta)-5 \beta]}{2(1+2 \alpha)(2-3 \beta)[2+2 \alpha(1-\beta)-3 \beta]} .
$$

Because $r>c, \beta<(1 / 2)$, and $\beta<((2 \alpha+2) /(2 \alpha+3))$, when $\beta<(4(1+\alpha) /(5+4 \alpha)), \quad w^{C N *}>w^{A N *}$, and when $\beta \geq(4(1+\alpha) /(5+4 \alpha)), \quad w^{C N *} \leq w^{A N *}$. Because $(4(1+\alpha) /(5+4 \alpha))<(1 / 2)$ and $\beta<(1 / 2), w^{C N *}>w^{A N *}$ is always satisfied.

$$
w^{C N *}-w^{B N *}=\frac{c+r+c \alpha+r \alpha-m^{C N *}}{2(1+\alpha)}-\frac{3 c+r+4 c \alpha+4 r \alpha}{4+8 \alpha} .
$$

Solving $w^{C N *}-w^{B N *}=0$, we can get a unique root $\{0\}$. $\left(\partial\left(w^{C N *}-w^{A N *}\right) /\left.\partial \beta\right|_{\beta=0}\right)=((r-c) /[8(1+\alpha)])>0$.

$\left(w^{C N *}-w^{B N *}\right)$ is an increasing function of $\beta$. Because $\beta>0$, $w^{C N *}>w^{B N *}$.

$$
\pi_{R}^{A N *}-\pi_{R}^{N N *}=\frac{(r-c)^{2}(1-\beta)^{2}}{2 t(2-3 \beta)}-\frac{(r-c)^{2}}{4 t}=\frac{(r-c)^{2} \beta(2 \beta-1)}{4 t(2-3 \beta)},
$$

because $\beta<(1 / 2), 2-3 \beta>0$, and $2 \beta-1<0, \pi_{R}^{A N *}<\pi_{R}^{N N *}$.

$$
\pi_{R}^{B N *}-\pi_{R}^{N N *}=\frac{(r-c)^{2}(1+\alpha)}{4 t(1+2 \alpha)}-\frac{(r-c)^{2}}{4 t}=-\frac{(r-c)^{2} \alpha}{4 t(1+2 \alpha)}<0,
$$

that is, $\pi_{R}^{B N *}<\pi_{R}^{N N *}$.

$$
\pi_{R}^{C N *}-\pi_{R}^{N N *}=\frac{2(r-c)^{2}(1+\alpha-\beta)^{2}-(r-c)^{2}(1+2 \alpha)[2+2 \alpha(1-\beta)-3 \beta]}{4 t(1+2 \alpha)[2+2 \alpha(1-\beta)-3 \beta]}=\frac{(r-c)^{2}[2 \alpha(1+\alpha)+\beta](2 \beta-1)}{4 t(1+2 \alpha)[2+2 \alpha(1-\beta)-3 \beta]},
$$

because $\beta<((2 \alpha+2) /(2 \alpha+3)), 2+2 \alpha(1-\beta)-3 \beta>0$. Because $\beta<(1 / 2), \pi_{R}^{C N *}<\pi_{R}^{N N *}$.

$$
\pi_{R}^{B N *}-\pi_{R}^{A N *}=\frac{(r-c)^{2}(1+\alpha)}{4 t(1+2 \alpha)}-\frac{(r-c)^{2}(1-\beta)^{2}}{2 t(2-3 \beta)}=\frac{(r-c)^{2}\left[(1+\alpha)(2-3 \beta)-2(1-\beta)^{2}(1+2 \alpha)\right]}{4 t(1+2 \alpha)(2-3 \beta)} .
$$


Let $\quad g_{5}(\beta)=(1+\alpha)(2-3 \beta)-2(1-\beta)^{2}(1+2 \alpha)$.

$\left(\partial^{2} g_{5}(\beta) / \partial \beta^{2}\right)=-4(1+2 \alpha)<0 . g_{5}(\beta)$ is a concave function

of $\beta$. Let $g_{5}(\beta)=0$, and we can get two roots:

$$
\begin{aligned}
& \left\{\frac{\left.1+5 \alpha-\sqrt{1-6 \alpha-7 \alpha^{2}}, \frac{1+5 \alpha+\sqrt{1-6 \alpha-7 \alpha^{2}}}{4+8 \alpha}\right\},}{4+8 \alpha}=\sqrt{(1+\alpha)(1-7 \alpha)}>0,\right. \\
& \frac{\left.\left(\partial g_{5}(\beta) / \partial \beta\right)\right|_{\beta=\left(\left(1+5 \alpha-\sqrt{1-6 \alpha-7 \alpha^{2}}\right) /(4+8 \alpha)\right)}}{\partial \beta}=-\sqrt{(1+\alpha)(1-7 \alpha)}<0, \quad \text { if } \alpha<\frac{1}{7} .
\end{aligned}
$$

Thus, when $\left(\left(1+5 \alpha-\sqrt{1-6 \alpha-} \quad 7 \alpha^{2}\right) /(4+8 \alpha)\right)<$ $\beta<\left(\left(1+5 \alpha+\sqrt{1-6 \alpha-7 \alpha^{2}}\right) /(4+8 \alpha)\right)$ and $\quad \alpha<1 / 7$, $g_{5}(\beta)>0$, then $\pi_{R}^{B N *}>\pi_{R}^{A N *}$. When $\beta \leq((1+5 \alpha-$

$$
\pi_{R}^{C N *}-\pi_{R}^{A N *}=\frac{(r-c)^{2}(1+\alpha-\beta)^{2}}{2 t(1+2 \alpha)[2+2 \alpha(1-\beta)-3 \beta]}-\frac{(r-c)^{2}(1-\beta)^{2}}{2 t(2-3 \beta)}=\frac{(r-c)^{2} \alpha(1-2 \beta)^{2}[-2(1+\alpha)+(2+\alpha) \beta]}{2 t(1+2 \alpha)[2+2 \alpha(1-\beta)-3 \beta](2-3 \beta)}
$$

Let $\left.g_{6}(\beta)=-2(1+\alpha)\right)+(2+\alpha) \beta$, which is a linear function of $\beta$. Let $g_{6}(\beta)=0$, and we can get a unique root $([2(1+\alpha)] /(2+\alpha))$. Because $\left(\partial g_{6}(\beta) / \partial \beta\right)=2+\alpha>0, g_{6}(\beta)$ is an increasing function of $\beta$. When $\beta<([2(1+\alpha)] /(2+\alpha))$,

$$
\pi_{R}^{C N *}-\pi_{R}^{B N *}=\frac{(r-c)^{2}(1+\alpha-\beta)^{2}}{2 t(1+2 \alpha)[2+2 \alpha(1-\beta)-3 \beta]}-\frac{(r-c)^{2}(1+\alpha)}{4 t(1+2 \alpha)}=\frac{(r-c)^{2} \beta\left(-1+\alpha+2 \alpha^{2}+2 \beta\right)}{4 t(1+2 \alpha)[2+2 \alpha(1-\beta)-3 \beta]} .
$$

Let $g_{7}(\beta)=-1+\alpha+2 \alpha^{2}+2 \beta$, which is a linear function of $\beta$. Let $g_{7}(\beta)=0$, and we can get a unique root $\left(1-\alpha-2 \alpha^{2}\right) / 2$. Because $\left(\partial g_{7}(\beta) / \partial \beta\right)=2>0, g_{7}(\beta)$ is an

increasing function of $\beta$. When $0<\beta<\left(1-\alpha-2 \alpha^{2}\right) / 2$, $g_{7}(\beta)<0, \quad$ then $\quad \pi_{R}^{C N *}<\pi_{R}^{B N *}$. $\left(\left(1-\alpha-2 \alpha^{2}\right) / 2\right) \leq \beta<(1 / 2), \pi_{R}^{C N *}>\pi_{R}^{B N *}$.

$$
\pi_{M}^{A N *}-\pi_{M}^{N N *}=\frac{(r-c)^{2}(1-\beta)^{2}}{2 t(2-3 \beta)^{2}}-\frac{(r-c)^{2}}{8 t}=\frac{(r-c)^{2}\left[4(1-\beta)^{2}-(2-3 \beta)^{2}\right]}{8 t(2-3 \beta)^{2}}=\frac{(r-c)^{2}(4-5 \beta) \beta}{8 t(2-3 \beta)^{2}}
$$

Because $\beta<(1 / 2), \quad 4-5 \beta>0, \quad \pi_{M}^{A N *}>\pi_{M}^{N N *} . \quad \pi_{M}^{B N *}-$

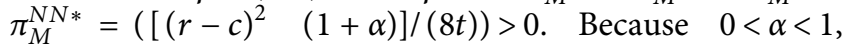
$\pi_{M}^{B N *}>\pi_{M}^{N N *}$.

$$
\pi_{M}^{C N *}-\pi_{M}^{N N *}=\frac{(r-c)^{2}(1+\alpha)(1+\alpha-\beta)^{2}}{2 t[2+2 \alpha(1-\beta)-3 \beta]^{2}}-\frac{(r-c)^{2}}{8 t}=\frac{(r-c)^{2} g_{8}(\beta)}{8 t[2+2 \alpha(1-\beta)-3 \beta]^{2}},
$$


where $g_{8}(\beta)=4(1+\alpha)(1+\alpha-\beta)^{2}-[2+2 \alpha(1-\beta)-3 \beta]^{2}$. Because $\left(\partial^{2} g_{8}(\beta) / \partial \beta^{2}\right)=-2[5+4 \alpha(2+\alpha)]<0, g_{8}(\beta)$ is a concave function of $\beta$. By solving $g_{8}(\beta)=0$, we can get two roots:

$$
\begin{aligned}
& \left\{\frac{2(1+\alpha)-2(1+2 \alpha)(1+\alpha) \sqrt{1+\alpha}}{5+4 \alpha(2+\alpha)}, \frac{2(1+\alpha)+2(1+2 \alpha)(1+\alpha) \sqrt{1+\alpha}}{5+4 \alpha(2+\alpha)}\right\}, \\
& \left.\frac{\partial g_{8}(\beta)}{\partial \beta}\right|_{\beta=([2(1+\alpha)-2(1+2 \alpha)(1+\alpha) \sqrt{1+\alpha}] /[5+4 \alpha(2+\alpha)])}=4(1+\alpha)(1+2 \alpha) \sqrt{1+\alpha}>0, \\
& \left.\frac{\partial g_{8}(\beta)}{\partial \beta}\right|_{\beta=([2(1+\alpha)+2(1+2 \alpha)(1+\alpha) \sqrt{1+\alpha}] /[5+4 \alpha(2+\alpha)])}=-4(1+\alpha)(1+2 \alpha) \sqrt{1+\alpha}<0 .
\end{aligned}
$$

Thus, when

$$
\frac{2(1+\alpha)-2(1+2 \alpha)(1+\alpha) \sqrt{1+\alpha}}{5+4 \alpha(2+\alpha)}<\beta<\frac{2(1+\alpha)+2(1+2 \alpha)(1+\alpha) \sqrt{1+\alpha}}{5+4 \alpha(2+\alpha)},
$$

$\pi_{M}^{C N *}>\pi_{M}^{N N *}$, or else $\pi_{M}^{C N *} \leq \pi_{M}^{N N *}$

$$
\pi_{M}^{B N *}-\pi_{M}^{A N *}=\frac{(r-c)^{2}(1+\alpha)}{8 t}-\frac{(r-c)^{2}(1-\beta)^{2}}{2 t(2-3 \beta)^{2}}=\frac{(r-c)^{2} g_{9}(\beta)}{8 t(2-3 \beta)^{2}}
$$

where $g_{9}(\beta)=(1+\alpha)(2-3 \beta)^{2}-4(1-\beta)^{2} . \quad\left(\partial^{2} g_{9}(\beta) /\right.$ $\left.\partial \beta^{2}\right)=2(5+9 \alpha)>0 . g_{9}(\beta)$ is a convex function of $\beta$. Let $g_{9}(\beta)=0$, and we can get two roots:

$$
\begin{aligned}
& \left\{\frac{2+6 \alpha-2 \sqrt{1+\alpha}}{5+9 \alpha}, \frac{2+6 \alpha+2 \sqrt{1+\alpha}}{5+9 \alpha}\right\}, \\
& \left.\frac{\partial \mathrm{g}_{9}(\beta)}{\partial \beta}\right|_{\beta=((2+6 \alpha-2 \sqrt{1+\alpha}) /(5+9 \alpha))}=-4 \sqrt{1+\alpha}<0, \\
& \left.\frac{\partial \mathrm{g}_{9}(\beta)}{\partial \beta}\right|_{\beta=((2+6 \alpha+2 \sqrt{1+\alpha}) /(5+9 \alpha))}=4 \sqrt{1+\alpha}>0 .
\end{aligned}
$$

Thus, when $((2+6 \alpha-2 \sqrt{1+\alpha})) /(5+9 \alpha)<\beta<((2+$ $6 \alpha+2 \sqrt{1+\alpha})) /(5+9 \alpha), g_{9}(\beta)<0$, then $\pi_{M}^{B N *}<\pi_{M}^{A N *}$, or else $\pi_{M}^{B N *} \geq \pi_{M}^{A N *}$.

$$
\pi_{M}^{C N *}-\pi_{M}^{A N *}=\frac{(r-c)^{2}(1+\alpha)(1+\alpha-\beta)^{2}}{2 t[2+2 \alpha(1-\beta)-3 \beta]^{2}}-\frac{(r-c)^{2}(1-\beta)^{2}}{2 t(2-3 \beta)^{2}}=\frac{(r-c)^{2} g_{10}(\beta)}{2 t[2+2 \alpha(1-\beta)-3 \beta]^{2}(2-3 \beta)^{2}}
$$


where

$$
\begin{aligned}
& g_{10}(\beta)=(1+\alpha)(1+\alpha-\beta)^{2}(2-3 \beta)^{2}-(1-\beta)^{2}[2+2 \alpha(1-\beta)-3 \beta]^{2} \\
& =[\sqrt{1+\alpha}(2-3 \beta)(1+\alpha-\beta)]^{2}-\{(1-\beta)[2+2 \alpha(1-\beta)-3 \beta]\}^{2} .
\end{aligned}
$$

Because $\beta<2 / 3,0<\beta<1$ and $\beta<((2 \alpha+2) /(2 \alpha+3))$, $\sqrt{1+\alpha}(2-3 \beta)(1+\alpha-\beta)>0$ and $(1-\beta)[2+2 \alpha(1-$ $\beta)-3 \beta]>0$. When $\sqrt{1+\alpha}(2-3 \beta)(1+\alpha-\beta)-(1-$

$$
\pi_{M}^{C N *}-\pi_{M}^{B N *}=\frac{(r-c)^{2}(1+\alpha)(1+\alpha-\beta)^{2}}{2 t[2+2 \alpha(1-\beta)-3 \beta]^{2}}-\frac{(r-c)^{2}(1+\alpha)}{8 t}=\frac{(r-c)^{2}(1+\alpha)\left\{4(1+\alpha-\beta)^{2}-[2+2 \alpha(1-\beta)-3 \beta]^{2}\right\}}{8 t[2+2 \alpha(1-\beta)-3 \beta]^{2}},
$$

where $g_{12}(\beta)=4(1+\alpha-\beta)^{2}-[2+2 \alpha(1-\beta)-3 \beta]^{2}$. Because $\left(\partial^{2} g_{12}(\beta) / \partial \beta^{2}\right)=-2(1+2 \alpha)(5+2 \alpha)<0, g_{12}(\beta)$ is a concave function of $\beta$. By solving $g_{12}(\beta)=0$, we can get too roots $\{0,(4(1+\alpha) /(5+2 \alpha))\}$, where $(4(1+\alpha) /(5+2 \alpha))>(1 / 2)$. $\left.\left(\partial g_{12}(\beta) / \partial \beta\right)\right|_{\beta=0}=4(1+\alpha)(1+2 \alpha)>0, \quad$ and $\left(\partial g_{12}(\beta) / \partial \beta\right)$ $\beta)[2+2 \alpha(1-\beta)-3 \beta]>0, g_{10}(\beta)>0$, then $\pi_{M}^{C N *}>\pi_{M}^{A N *}$, or else $\pi_{M}^{C N *} \leq \pi_{M}^{A N *}$. $\left.\right|_{\beta=(4(1+\alpha) /(5+2 \alpha))}=-4(1+\alpha)(1+2 \alpha)<0 . \quad$ Thus, when $0<\beta<(1 / 2), \pi_{M}^{C N *}>\pi_{M}^{B N *}$.

\section{E. Proof of Proposition 6}

$$
m^{A E *}-m^{N E *}=\frac{(\mathrm{d} t+2 r-2 c)(1+2 \alpha)}{3(2+3 \alpha)}-\frac{2 r+\mathrm{d} t-2 c}{6}=\frac{[\mathrm{d} t+2(r-c)] \alpha}{6(2+3 \alpha)}>0 .
$$

Thus, $m^{A E *}>m^{N E *}$.

$$
m^{B E *}-m^{N E *}=\frac{(\mathrm{d} t+2 r-2 c)(1-\beta)}{6(1-2 \beta)}-\frac{2 r+\mathrm{d} t-2 c}{6}=\frac{(2 r+\mathrm{d} t-2 c) \beta}{6(1-2 \beta)}>0 .
$$

Thus, $m^{B E *}>m^{N E *}$.

$$
m^{C E *}-m^{N E *}=\frac{(\mathrm{d} t+2 r-2 c)(1+2 \alpha)(1-\beta)^{2}}{3(1-2 \beta)[2+3 \alpha-2(1+\alpha) \beta]}-\frac{2 r+\mathrm{d} t-2 c}{6}=\frac{(2 r+\mathrm{d} t-2 c)[\alpha+2(1-\beta) \beta]}{6(1-2 \beta)(2+3 \alpha-2(1+\alpha) \beta)}
$$

Because $\beta<(1 / 2), 1-2 \beta>0,2+3 \alpha-2(1+\alpha) \beta>0$ and $\alpha+2(1-\beta) \beta>0$. Thus, $m^{\mathrm{CE*}}-m^{N E *}>0$; then, $m^{C E *}>m^{N E *}$.

$$
m^{B E *}-m^{A E *}=\frac{(\mathrm{d} t+2 r-2 c)(1-\beta)}{6(1-2 \beta)}-\frac{(\mathrm{d} t+2 r-2 c)(1+2 \alpha)}{3(2+3 \alpha)}=\frac{(\mathrm{d} t+2 r-2 c)[(2+5 \alpha)) \beta-\alpha]}{6(1-2 \beta)(2+3 \alpha)} .
$$


When $\quad \beta>(\alpha /(5 \alpha+2)), \quad m^{B E *}-m^{A E *}>0$, i.e., that $\beta<(1 / 2)$. Thus, when $\quad(\alpha /(5 \alpha+2))<\beta<(1 / 2)$, $m^{B E *}>m^{A E *}$. When $\beta \leq(\alpha /(5 \alpha+2)), m^{B E *} \leq m^{A E *}$. Note $\quad m^{B E *}>m^{A E *}$. When $0<\beta \leq(\alpha /(5 \alpha+2)), m^{B E *} \leq m^{A E *}$.

$$
m^{C E *}-m^{A E *}=\frac{(\mathrm{d} t+2 r-2 c)(1+2 \alpha)(1-\beta)^{2}}{3(1-2 \beta)[2+3 \alpha-2(1+\alpha) \beta]}-\frac{(\mathrm{d} t+2 r-2 c)(1+2 \alpha)}{3(2+3 \alpha)}=\frac{(\mathrm{d} t+2 r-2 c)(1+2 \alpha) \beta[2(1+\alpha)-(2+\alpha) \beta]}{3(1-2 \beta)[2+3 \alpha-2(1+\alpha) \beta](2+3 \alpha)}
$$

Because $\beta<(1 / 2), 1-2 \beta>0,2(1+\alpha)-(2+\alpha) \beta>0$ and $2+3 \alpha-2(1+\alpha) \beta>0$. Thus, $m^{C E *}>m^{A E *}$.

$$
m^{C E *}-m^{B E *}=\frac{(\mathrm{d} t+2 r-2 c)(1+2 \alpha)(1-\beta)^{2}}{3(1-2 \beta)[2+3 \alpha-2(1+\alpha) \beta]}-\frac{(\mathrm{d} t+2 r-2 c)(1-\beta)}{6(1-2 \beta)}=\frac{(1-\beta)(\mathrm{d} t+2 r-2 c) \alpha(1-2 \beta)}{6(1-2 \beta)[2+3 \alpha-2(1+\alpha) \beta]}
$$

Because $\beta<(1 / 2), 1-2 \beta>0$, and $2+3 \alpha-2(1+\alpha) \beta>0$, $m^{C E *}-m^{B E *}>0$, i.e., $m^{C E *}>m^{B E *}$.

$$
w^{A E *}-w^{N E *}=\frac{(2 r+\mathrm{d} t)(4+5 \alpha)+2 c(8+13 \alpha)}{12(2+3 \alpha)}-\frac{2 r+\mathrm{d} t+4 c}{6}=-\frac{(2 r+\mathrm{d} t-2 c) \alpha}{12(2+3 \alpha)}<0
$$

Thus, $w^{A E *}<w^{N E *}$.

$$
\begin{aligned}
w^{B E *}-w^{N E *} & =\frac{(2 r+\mathrm{d} t)(1-3 \beta)+c(4-6 \beta)}{6(1-2 \beta)}-\frac{2 r+\mathrm{d} t+4 c}{6}=\frac{(2 r+\mathrm{d} t)(1-3 \beta)+c(4-6 \beta)}{6(1-2 \beta)}-\frac{(2 r+\mathrm{d} t+4 c)(1-2 \beta)}{6(1-2 \beta)} \\
& =-\frac{(2 r+\mathrm{d} t-4 c) \beta}{6(1-2 \beta)}<0
\end{aligned}
$$

Thus, $w^{B E *}<w^{N E *}$.

$$
w^{C E *}-w^{N E *}=-\frac{(2 r+\mathrm{d} t-2 c)\{4(1-\beta) \beta+\alpha[1+4(1-\beta) \beta]\}}{12(1-2 \beta)[2+3 \alpha-2(1+\alpha) \beta]}<0
$$

and thus $w^{C E *}<w^{N E *}$.

$$
w^{B E *}-w^{A E *}=\frac{(2 r+\mathrm{d} t)(1-3 \beta)+c(4-6 \beta)}{6(1-2 \beta)}-\frac{(2 r+\mathrm{d} t)(4+5 \alpha)+2 c(8+13 \alpha)}{12(2+3 \alpha)}=-\frac{(2 r+\mathrm{d} t-2 c)[4 \beta-\alpha(1-8 \beta)]}{12(1-2 \beta)(2+3 \alpha)} .
$$


Because $\quad \beta<(1 / 2), \quad 1-2 \beta>0, \quad$ and when $\beta>(\alpha /[4(1+2 \alpha)]), 4 \beta-\alpha(1-8 \beta)>0, w^{B E *}<w^{A E *}$, or else $w^{B E *} \geq w^{A E *}$. Note that $(\alpha / 4(1+2 \alpha))<(1 / 2)$.

$$
w^{C E *}-w^{A E *}=\frac{(2 r+\mathrm{d} t-2 c)(1+2 \alpha) \beta[-4-5 \alpha+4(1+\alpha) \beta]}{6(2+3 \alpha)(1-2 \beta)[2+3 \alpha-2(1+\alpha) \beta]}=\frac{(2 r+\mathrm{d} t-2 c)(1+2 \alpha) \beta[-4(1-\beta)-4 \alpha(1-\beta)-\alpha]}{6(2+3 \alpha)(1-2 \beta)[2+3 \alpha-2(1+\alpha) \beta]}<0 .
$$

Thus, $w^{C E *}<w^{A E *}$.

Thus, $w^{C E *}<w^{B E *}$.

$$
w^{C E *}-w^{B E *}=-\frac{(2 r+\mathrm{d} t-2 c) \alpha}{12[2+3 \alpha-2(1+\alpha) \beta]}<0 .
$$

$$
\pi_{R}^{A E *}-\pi_{R}^{N E *}=\frac{(2 r+\mathrm{d} t-2 c)^{2}\left(1-2 \alpha-5 \alpha^{2}\right)}{24 t(2+3 \alpha)}-\frac{(2 r+\mathrm{d} t-2 c)^{2}}{48 t}=-\frac{(2 r+\mathrm{d} t-2 c)^{2}\left(7 \alpha+10 \alpha^{2}\right)}{48 t(2+3 \alpha)}<0 .
$$

Thus, $\pi_{R}^{A E *}<\pi_{R}^{N E *}$.

$$
\pi_{R}^{B E *}-\pi_{R}^{N E *}=\frac{(2 r+\mathrm{d} t-2 c)^{2}(1-\beta)}{48 t(1-2 \beta)}-\frac{(2 r+\mathrm{d} t-2 c)^{2}}{48 t}=\frac{(2 r+\mathrm{d} t-2 c)^{2} \beta}{48 t(1-2 \beta)}>0 .
$$

Thus, $\pi_{R}^{B E *}>\pi_{R}^{N E *}$.

$$
\pi_{R}^{C E *}-\pi_{R}^{N E *}=\frac{(2 r+\mathrm{d} t-2 c)^{2}}{48 t(1-2 \beta)[2+3 \alpha-2(1+\alpha) \beta]} V_{1}(\beta),
$$

$$
\begin{aligned}
& \hat{\beta}_{1}=\frac{1-\sqrt{(1+2 \alpha)^{2}(1+4 \alpha)(1+6 \alpha)}+2 \alpha(7+8 \alpha)}{2+4 \alpha(5+4 \alpha)}, \\
& \hat{\beta}_{2}=\frac{1+\sqrt{(1+2 \alpha)^{2}(1+4 \alpha)(1+6 \alpha)}+2 \alpha(7+8 \alpha)}{2+4 \alpha(5+4 \alpha)} .
\end{aligned}
$$

where cause $\left(\partial^{2} V_{1}(\beta) / \partial \beta^{2}\right)=-4-40 \alpha-32 \alpha^{2}<0, V_{1}(\beta)$ is concave function of $\beta$. By solving $V_{1}(\beta)=0$ for $\beta$, we can get $\pi_{R}^{C E *} \leq \pi_{R}^{N E *}$. two roots $\left\{\widehat{\beta}_{1}, \widehat{\beta}_{2}\right\}$, where

$$
\begin{aligned}
\pi_{R}^{B E *}-\pi_{R}^{A E *} & =\frac{(2 r+\mathrm{d} t-2 c)^{2}(1-\beta)}{48 t(1-2 \beta)}-\frac{(2 r+\mathrm{d} t-2 c)^{2}\left(1-2 \alpha-5 \alpha^{2}\right)}{24 t(2+3 \alpha)} \\
& =\frac{(2 r+\mathrm{d} t-2 c)^{2}}{48 t(1-2 \beta)(2+3 \alpha)}\left[(1-\beta)(2+3 \alpha)-2\left(1-2 \alpha-5 \alpha^{2}\right)(1-2 \beta)\right] .
\end{aligned}
$$

When $\beta<\left(\left(7 \alpha+10 \alpha^{2}\right) /\left(20 \alpha^{2}+11 \alpha-2\right)\right), \pi_{R}^{B E *}>\pi_{R}^{A E *}$, or else $\pi_{R}^{B E *} \leq \pi_{R}^{A E *}$.

$$
\pi_{R}^{C E *}-\pi_{R}^{A E *}=\frac{(2 r+\mathrm{d} t-2 c)^{2}(1+2 \alpha)^{2} \beta[2(1+\alpha)-(2+\alpha) \beta]}{24 t(2+3 \alpha)(1-2 \beta)[2+3 \alpha-2(1+\alpha) \beta]} .
$$

When $\beta<([2(1+\alpha)] /(2+\alpha)), \pi_{R}^{C E *}>\pi_{R}^{A E *}$, or else $\pi_{R}^{C E *} \leq \pi_{R}^{A E *}$. Note that $([2(1+\alpha)] /(2+\alpha))>(1 / 2)$ and $\beta<(1 / 2)$; thus, $\pi_{R}^{C E *}>\pi_{R}^{A E *}$.

$$
\pi_{R}^{C E *}-\pi_{R}^{B E *}=\frac{(2 r+\mathrm{d} t-2 c)^{2} \alpha V_{2}(\beta)}{48 t(1-2 \beta)[2+3 \alpha-2(1+\alpha) \beta]} .
$$


Let $\quad V_{2}(\beta)=(1-\beta)(18 \beta-7)+2 \alpha[8(2-\beta) \beta-5]$. $\left(\partial^{2} V_{2}(\beta) / \partial \beta^{2}\right)=-36-32 \alpha<0 . V_{2}(\beta)$ is a concave function of $\beta$. By solving $V_{2}(\beta)=0$, we can get two roots $\left\{\widehat{\beta}_{3}, \widehat{\beta}_{4}\right\}$, where

$$
\begin{aligned}
& \widehat{\beta}_{3}=\frac{25+32 \alpha-\sqrt{121+48 \alpha(9+8 \alpha)}}{4(9+8 \alpha)}, \\
& \widehat{\beta}_{4}=\frac{25+32 \alpha+\sqrt{121+48 \alpha(9+8 \alpha)}}{4(9+8 \alpha)} .
\end{aligned}
$$

Because $V_{2}(0)=-7-10 \alpha<0$ and $V_{2}(1 / 2)=1+2 \alpha>0$, there is a unique root between the interval $(0,1 / 2)$. Thus, when $0<\beta<\widehat{\beta}_{3}, \quad \pi_{R}^{C E *}<\pi_{R}^{B E *}$. When $\widehat{\beta}_{3} \leq \beta<1 / 2$, $\pi_{R}^{C E *} \geq \pi_{R}^{B E *}$.

$$
\pi_{M}^{A E *}-\pi_{M}^{N E *}=\frac{(2 r+\mathrm{d} t-2 c)^{2}\left(9+26 \alpha+19 \alpha^{2}\right)}{24 t(2+3 \alpha)^{2}}-\frac{3(2 r+\mathrm{d} t-2 c)^{2}}{32 t}=-\frac{(2 r+\mathrm{d} t-2 c)^{2}}{96 t(2+3 \alpha)^{2}}(4+5 \alpha) \alpha<0 .
$$

Thus, $\pi_{M}^{A E *}<\pi_{M}^{N E *}$.

Thus, $\pi_{M}^{B E *}<\pi_{M}^{N E *}$.

$$
\pi_{M}^{B E *}-\pi_{M}^{N E *}=\frac{3(2 r+\mathrm{d} t-2 c)^{2}(1-\beta)}{32 t}-\frac{3(2 r+\mathrm{d} t-2 c)^{2}}{32 t}<0 .
$$

$$
\pi_{M}^{C E *}-\pi_{M}^{N E *}=-\frac{(2 r+\mathrm{d} t-2 c)^{2}}{96 t[2+3 \alpha-2(1+\alpha) \beta]^{2}}\left\{36(1-\beta)^{2} \beta+4 \alpha(1-\beta)[1+8 \beta(3-2 \beta)]+\alpha^{2}\left\{5+4 \beta V_{5}(\beta)\right\}\right\}
$$

where $\quad V_{5}(\beta)=16+\beta(-23+8 \beta)$. Because $\left(\partial^{2} V_{5}(\beta) / \partial \beta^{2}\right)=16>0, V_{5}(\beta)$ is a convex function of $\beta$. By solving $V_{5}(\beta)=0$, we can get two roots

$\{((23-\sqrt{17}) / 16),(23+\sqrt{17}) / 16\}$, where both roots are greater than $(1 / 2)$. Thus, in the interval $\beta \in(0,1 / 2)$, $V_{5}(\beta)>0$. As a result, $\pi_{M}^{C E *}<\pi_{M}^{N E *}$.

$$
\pi_{M}^{B E *}-\pi_{M}^{A E *}=\frac{3(2 r+\mathrm{d} t-2 c)^{2}(1-\beta)}{32 t}-\frac{(2 r+\mathrm{d} t-2 c)^{2}\left(9+26 \alpha+19 \alpha^{2}\right)}{24 t(2+3 \alpha)^{2}}=\frac{(2 r+\mathrm{d} t-2 c)^{2}}{96 t(2+3 \alpha)^{2}} V_{6}(\beta),
$$

where $\quad V_{6}(\beta)=9(1-\beta)(2+3 \alpha)^{2}-4\left(9+26 \alpha+19 \alpha^{2}\right)$. $V_{6}(\beta)$ is a linear function of $\beta$. Because $\left(\partial V_{6}(\beta) / \partial \beta\right)=-9(2+3 \alpha)^{2}<0, \quad V_{6}(\beta)$ is a decreasing function of $\beta$. Solving $V_{6}(\beta)=0$ for $\beta$, we can get a unique root $\left(\left(4 \alpha+5 \alpha^{2}\right) /\left[9(2+3 \alpha)^{2}\right]\right)<(1 / 2)$. Thus, when $0<\beta<\left(\left(4 \alpha+5 \alpha^{2}\right) /\left[9(2+3 \alpha)^{2}\right]\right), \quad \pi_{M}^{B E *}>\pi_{M}^{A E *}$; $\left(\left(4 \alpha+5 \alpha^{2}\right) /\left[9(2+3 \alpha)^{2}\right]\right) \leq \beta<1 / 2, \pi_{M}^{B E *} \leq \pi_{M}^{A E *}$.

$$
\begin{aligned}
U_{M}^{C E *}-\pi_{M}^{A E *} & =\frac{(2 r+\mathrm{d} t-2 c)^{2}(1-\beta) G}{24 t[2+3 \alpha-2(1+\alpha) \beta]^{2}}-\frac{(2 r+\mathrm{d} t-2 c)^{2}\left(9+26 \alpha+19 \alpha^{2}\right)}{24 t(2+3 \alpha)^{2}} \\
& =\frac{(2 r+\mathrm{d} t-2 c)^{2}}{24 t[2+3 \alpha-2(1+\alpha) \beta]^{2}(2+3 \alpha)^{2}}\left\{(1-\beta)(2+3 \alpha)^{2} G-\left(9+26 \alpha+19 \alpha^{2}\right)[2+3 \alpha-2(1+\alpha) \beta]^{2}\right\}
\end{aligned}
$$

When $\quad(1-\beta)(2+3 \alpha)^{2} G^{-} \quad\left(9+26 \alpha+19 \alpha^{2}\right)[2+3 \alpha-$ $2(1+\alpha) \beta]^{2}>0, U_{M}^{C E *}>\pi_{M}^{A E *}$, or else $U_{M}^{C E *} \leq \pi_{M}^{A E *}$.

$$
\pi_{M}^{C E *}-\pi_{M}^{B E *}=\frac{(2 r+\mathrm{d} t-2 c)^{2} \alpha(1-\beta)(1-2 \beta) V_{7}(\beta)}{96 t[2+3 \alpha-2(1+\alpha) \beta]^{2}}
$$


where $V_{7}(\beta)=-4-5 \alpha+2(2+\alpha) \beta$. Because $\left(\partial V_{7}(\beta) /\right.$ $\partial \beta)=2(2+\alpha)>0, V_{7}(\beta)$ is an increasing function of $\beta$. By solving $V_{7}(\beta)=0$, we can get a unique root $((4+$ $5 \alpha) /[2(2+\alpha)])$. Thus, when $\beta<((4+5 \alpha) /[2(2+\alpha)])$, $\pi_{M}^{C E *}<\pi_{M}^{B E *}$. When $\beta \geq((4+5 \alpha) /[2(2+\alpha)]), \pi_{M}^{C E *} \geq \pi_{M}^{B E *}$. Note that $((4+5 \alpha) /[2(2+\alpha)])>1$ and $\beta<(1 / 2)$, and thus $\pi_{M}^{C E *}<\pi_{M}^{B E *}$ are always satisfied.

\section{Data Availability}

All data generated or analyzed during this study are included within this article. The author is willing to share the implementation scripts in the form of some MATHEMATICA nb-files with the interested reader.

\section{Conflicts of Interest}

The author declares that there are no conflicts of interest regarding the publication of this paper.

\section{Acknowledgments}

This study was supported by the National Natural Science Foundation of China (71601111, 71774109, and 71702101) and Natural Science Foundation of Shanghai (18ZR1416900).

\section{References}

[1] B. Z. Niu, L. Chen, and F. F. Xie, "Production outsourcing for limited-edition luxury goods with consideration of consumers' origin preferences," Transportation Research E-Logistics and Transportation Review, vol. 140, Article ID 101975, 2020.

[2] B. Hu, Y. Mai, and S. Pekeč, "Managing innovation spillover in outsourcing," Production and Operations Management, vol. 29, no. 10, p. 2252, 2020.

[3] J. Heydari, K. Govindan, H. R. E. Nasab, and A. A. Taleizadeh, "Coordination by quantity flexibility contract in a two-echelon supply chain system: effect of outsourcing decisions," International Journal of Production Economics, vol. 225, Article ID 107586, 2020.

[4] Q. Cui, "Quality investment, and the contract manufacturer's encroachment," European Journal of Operational Research, vol. 279, no. 2, pp. 407-418, 2019.

[5] J. Shi, "Contract manufacturer's encroachment strategy and quality decision with different channel leadership structurers," Computers \& Industrial Engineering, vol. 137, Article ID 106078, 2019.

[6] J. Chen, L. Liang, and D.-q. Yao, "Factory encroachment and channel selection in an outsourced supply chain," International Journal of Production Economics, vol. 215, pp. 73-83, 2019.

[7] B. Niu, Y. Wang, and P. Guo, "Equilibrium pricing sequence in a co-opetitive supply chain with the ODM as a downstream rival of its OEM," Omega, vol. 57, pp. 249-270, 2015.

[8] E. Fehr and K. M. Schmidt, "A theory of fairness, competition, and cooperation," The Quarterly Journal of Economics, vol. 114, no. 3, pp. 817-868, 1999.

[9] X. P. Zhen, D. Shi, S. B. Tsai, and W. Wang, "Pricing decisions of a supply chain with multichannel retailer under fairness concerns," Mathematical Problems in Engineering, vol. 2019, Article ID 9547302, 2019.
[10] A. Adhikari and A. Bisi, "Collaboration, bargaining, and fairness concern for a green apparel supply chain: an emerging economy perspective," Transportation Research Part E: Logistics and Transportation Review, vol. 135, Article ID 101863, 2020.

[11] N. Amrouche and G. Zaccour, "Shelf-space allocation of national and private brands," European Journal of Operational Research, vol. 180, no. 2, pp. 648-663, 2007.

[12] S. Choi and K. Fredj, "Price competition and store competition: store brands vs. national brand," European Journal of Operational Research, vol. 225, no. 1, pp. 166-178, 2013.

[13] B. Liao, C. A. Yano, and M. Trivedi, "Optimizing store-brand quality: impact of choice of producer and channel price leadership," Production and Operations Management, vol. 29, no. 1, pp. 118-137, 2020.

[14] L. Wang, J. Chen, and H. Song, "Manufacturer's channel strategy with retailer's store brand," International Journal of Production Research, vol. 1, 2020.

[15] Z. S. Huang and T. Feng, "Money-back guarantee and pricing decision with retailer's store brand," Journal of Retailing and Consumer Services, vol. 52, Article ID 101897, 2020.

[16] W.-y. K. Chiang, D. Chhajed, and J. D. Hess, "Direct marketing, indirect profits: a strategic analysis of dual-channel supply-chain design," Management Science, vol. 49, no. 1, pp. 1-20, 2003.

[17] A. A. Tsay and N. Agrawal, "Channel dynamics under price and service competition," Manufacturing \& Service Operations Management, vol. 2, no. 4, pp. 372-391, 2000.

[18] A. Arya, B. Mittendorf, and D. E. M. Sappington, "The bright side of supplier encroachment," Marketing Science, vol. 26, no. 5, pp. 651-659, 2007.

[19] K. Matsui, "Optimal bargaining timing of a wholesale price for a manufacturer with a retailer in a dual-channel supply chain," European Journal of Operational Research, vol. 287, no. 1, pp. 225-236, 2020.

[20] D. E. Mills, "Why retailers sell private labels," Journal of Economics, vol. 4, no. 3, pp. 509-528, 1995.

[21] R. Sethuraman, "Assessing the external validity of analytical results from national brand and store brand competition models," Marketing Science, vol. 28, no. 4, pp. 759-781, 2009.

[22] J. Ru, R. Shi, and J. Zhang, "Does a store brand always hurt the manufacturer of a competing national brand?" Production and Operations Management, vol. 24, no. 2, pp. 272-286, 2015.

[23] K. Pauwels and S. Srinivasan, "Who benefits from store brand entry?” Marketing Science, vol. 23, no. 3, pp. 364-390, 2004.

[24] T. T. Li, J. X. Xie, X. B. Zhao, and J. F. Tang, "On supplier encroachment with retailer's fairness concerns," Computers \& Industrial Engineering, vol. 98, pp. 499-512, 2016.

[25] J. Shi, "Contract manufacturer's encroachment strategy considering advertising effort," Journal of Service and Management, vol. 13, pp. 229-243, 2019.

[26] Z. M. Guan, T. Ye, and R. Yin, "Channel coordination under Nash bargaining fairness concerns in differential games of goodwill accumulation," European Journal of Operational Research, vol. 285, pp. 916-930, 2020.

[27] K. W. Pan, Z. B. Cui, A. Xing, and Q. H. Lu, "Impact of fairness concern on retailer-dominated supply chain," Computers \& Industrial Engineering, vol. 139, Article ID 106209, 2020.

[28] Y. Liu, D. D. Wang, and Q. Xu, “A supply chain coordination mechanism with suppliers' effort performance level and fairness concern," Journal of Retailing and Consumer Services, vol. 53, Article ID 101950, 2020. 
[29] H. Y. Zhang, Z. Zhang, X. J. Pu, and Y. H. Li, "Green manufacturing strategy considering retailers' fairness concerns," Sustainability, vol. 11, no. 17, Article ID 4646, 2019.

[30] L. H. Zhang, H. Zhou, Y. Y. Liu, and R. Lu, "Optimal environmental quality and price with consumer environmental awareness and retailer's fairness concerns in supply chain," Journal of Cleaner Production, vol. 213, pp. 1063-1079, 2019.

[31] A. Sharma, G. Dwivedi, and A. Singh, "Game-theoretic analysis of a two-echelon supply chain with option contract under fairness concerns," Computers \& Industrial Engineering, vol. 137, Article ID 106096, 2019.

[32] W. H. Liu, D. Wang, X. R. Shen, X. Y. Yan, and W. Wei, "The impacts of distributional and peer-induced fairness concerns on the decision-making of order allocation in logistics service supply chain," Transportation Research Part E: Logistics and Transportation Review, vol. 116, pp. 102-122, 2018.

[33] C. Liu and W. D. Chen, "Decision making in green supply chains under the impact of the stochastic and multiple-variable dependent reference point," Transportation Research Part E: Logistics and Transportation Review, vol. 128, pp. 443-469, 2019.

[34] Q. Q. Li, T. J. Xiao, and Y. Z. Qiu, "Price and carbon emission reduction decisions and revenue-sharing contract considering fairness concerns," Journal of Cleaner Production, vol. 190, pp. 303-314, 2018.

[35] Z. S. Huang and T. Feng, "Impact of fairness concern on store brand strategy of powerful retailer," Application Research of Computer, vol. 37, no. 3, pp. 789-793, 2018.

[36] Q. H. Li and B. Li, "Dual-channel supply chain equilibrium problems regarding retail services and fairness concerns," Applied Mathematical Modelling, vol. 40, pp. 7349-7367, 2016.

[37] M. Arshad, Q. S. Khalid, J. Lloret, and A. Leon, "An efficient approach for coordination of dual-channel closed-loop supply chain management," Sustainability, vol. 10, no. 10, Article ID 3433, 2018.

[38] H. Hotelling, "Stability in competition," Economic Journal, vol. 39, pp. 41-57, 1929.

[39] N. Xia and S. Rajagopalan, "Standard vs. custom products: variety, leadtime, and price competition," Marketing Science, vol. 28, no. 5, pp. 887-900, 2009.

[40] Y. Xu, H. Gurnani, and R. Desiraju, "Strategic supply chain structurer design for a proprietary component manufacturer," Production and Operations Management, vol. 19, no. 4, pp. 371-389, 2010.

[41] H. Wong and D. Eyers, "An analytical framework for evaluating the value of enhanced customization: an integrated operations-marketing perspective," International Journal of Production Research, vol. 49, no. 1, pp. 5770-5800, 2011.

[42] T. J. Xiao, J. Shi, and G. H. Chen, "Price and leadtime competition, and coordination for make-to-order supply chains," Computers \& Industrial Engineering, vol. 68, pp. 23-34, 2014.

[43] T. F. Nie and S. F. Du, "Dual-fairness supply chain with quantity discount contract," European Journal of Operational Research, vol. 258, no. 2, pp. 491-500, 2017.

[44] H. T. Cui, J. S. Raju, and Z. J. Zhang, "Fairness and channel coordination," Management Science, vol. 53, no. 8, pp. 1303-1314, 2007. 